\title{
Modelling dynamics of marathons - A mixture model approach
}

\section{DOI:}

10.1016/j.physa.2019.04.034

\section{Document Version}

Accepted author manuscript

Link to publication record in Manchester Research Explorer

\section{Citation for published version (APA):}

Kwong, H. S., \& Nadarajah, S. (2019). Modelling dynamics of marathons - A mixture model approach. Physica A: Statistical Mechanics and its Applications, [120798]. https://doi.org/10.1016/j.physa.2019.04.034

\section{Published in:}

Physica A: Statistical Mechanics and its Applications

\section{Citing this paper}

Please note that where the full-text provided on Manchester Research Explorer is the Author Accepted Manuscript or Proof version this may differ from the final Published version. If citing, it is advised that you check and use the publisher's definitive version.

\section{General rights}

Copyright and moral rights for the publications made accessible in the Research Explorer are retained by the authors and/or other copyright owners and it is a condition of accessing publications that users recognise and abide by the legal requirements associated with these rights.

\section{Takedown policy}

If you believe that this document breaches copyright please refer to the University of Manchester's Takedown Procedures [http://man.ac.uk/04Y6Bo] or contact uml.scholarlycommunications@manchester.ac.uk providing relevant details, so we can investigate your claim.

\section{OPEN ACCESS}




\title{
Modelling dynamics of marathons - A mixture model approach
}

\author{
by \\ Hok Shing Kwong and Saralees Nadarajah \\ School of Mathematics, University of Manchester, Manchester M13 9PL, UK
}

\begin{abstract}
In this paper, statistical properties of marathon dynamics are studied. We find that changes of velocity in marathons follow an unconventional mechanism; in which the log-change of velocity is highly dependent on current velocity with a complex relationship. The conditional distributions of log-change of velocity exhibit patterns of varying means, variances, and skewnesses; as such, the overall velocity distributions are also found to have departed from Gaussian. We illustrate the mechanism with a finite mixture of generalized linear regressions with varying weights and skew normal errors; and we show that the completion time distribution can be approximated by skewed distributions.
\end{abstract}

Keywords: Finite mixture model; Generalized linear model; HMC; Logistic regression; MCMC; Skew exponential power distribution; Skew normal distribution; Velocity distribution

\section{Introduction}

Human physical abilities are the foundation of most sports. Many of these abilities can be demonstrated in the sports of athletics, for example, running, jumping and throwing. Statistical properties of these abilities of human beings are useful in many fields such as physiology, sports science and biological science. However, the majority of sports datasets contains only information of professional athletes, which can not reflect abilities of ordinary people; at the same time, it is difficult and costly to collect the same type of data in a large scale from ordinary people. In contrast, results from marathon races contain information of a wider spectrum of people, from which statistical properties of these results can better reflect physical abilities of human beings as a whole.

Statistical analyses of marathon results have been carried out. Some of the most recent papers are as follows. Lin and Meng (2018) considered several datasets from the largest city marathons in the world, they found that distributions of marathon interval completion time change from Gaussian distribution to log-normal distribution and back to Gaussian distribution along the marathon. Rodriguez et al. (2014) modeled the dynamics of marathon runner packs with a convection diffusion process. Lehto (2016) analysed the effects of age on marathon finishing times. Alvarez-Ramirez and Rodriguez (2006) analysed the scaling properties of marathon races, they found that there is a power law relationship between ranking and average velocity of high performance marathon runners, and this relationship becomes linear for average marathon runners. Alvarez-Ramirez et al. (2007) analysed arrival sequences in marathons, and found a time clustering phenomena.

Due to the central limit theorem, many human variables such as height can be approximated by the Gaussian distribution; however, this assumption may not hold for running velocity. As presented in Lin and Meng (2018), average velocities were found switching between Gaussian and log-normal distributions at different stage of a marathon. The cause of this phenomenon has not been studied; in view of this, we believe a study on marathon dynamics would lead to a better understanding of 
this phenomenon. Moreover, it is reasonable to assume that the change of shape of the distribution is gradual; therefore it is desirable to describe such shape changes via parameterisation within a class of distributions. Overall, there are two objectives in this paper: the first objective is to identify a suitable class of distributions for interval completion times; the second objective is to develop a model that describes and estimates how velocity changes.

The remainder of this paper is organised as follows. Section 2 describes the datasets to be used and their statistical properties. Section 3 describes the reasons for the choice of distributions. It also presents illustrations and comparisons of the proposed distributions. Section 4 describes a model to estimate changes of average velocity in marathons. Conclusions and remarks are given in Section 5 .

\section{Data description}

For the first part of the analyses, we consider results of four city marathons in 2017: New York City marathon, Boston marathon, Berlin marathon and Chicago marathon, which are arguably the most competitive marathons in the world held annually. Entries of these marathons can be divided generally into two groups: a) Time qualifying group, and b) Non time qualifying group. Some qualifying times are age and gender dependent, and are different for each marathon. The qualifying times of these marathons in 2017 for men aged between 25 to 29 (25-29M) are shown in Table 1. For some marathons, for example, the Boston marathon, not all qualifiers are guaranteed a spot. Cut off times are adjusted to limit the number of qualifying entries every year after receiving all applications. For non-qualifiers, one can gain a spot via various entries, for example, lottery type entry or charity/club entry. We assume non qualifying entries are indiscriminate of one's physical abilities. It is clear that there exists at least two sub-groups of runners of different physical capabilities. The differences in statistical properties can not be determined directly from the data since there is no information about entry method in the datasets. The use of mixture model is a natural solution to draw statistical inference of each sub-group.

For the second part of the analyses, we consider results of Chicago marathon from 2011 to 2018, during when the interval completion times were recorded every 5 kilometers. A total number of 281,582 finishers was recorded. Among these data points, 29,426 contained estimates of interval completion times, which were estimated with the assumption of constant velocity. These data points are excluded from our analyses. With a larger number of data points, better inference can be drawn, especially on the slowest group of runners, which exhibits different behaviour than the major group of runners.

\begin{tabular}{cccc} 
Marathon & Times (minute) & Number of finishers & Number of qualifying finishers \\
\hline New York City & 173 & 2629 & 107 \\
Boston & 185 & 1094 & 385 \\
Berlin & 165 & 2343 & 113 \\
Chicago & 195 & 2558 & 315 \\
\hline
\end{tabular}

Table 1: Qualifying times for men aged between 25-29 in 2017.

Each dataset contains thirteen variables: age group, gender, finishing time $t_{f}$, half finishing time $t_{h}$, and eight interval completion times recorded every five kilometers $t_{5}, \ldots, t_{40}$. The fastest 
running gender age group (25-29M) of each marathon is used for the first part of the analyses in this paper for illustration purposes. Figure 1 plots the densities of $\log$ finishing times $\log t_{f}$ of $25-29 \mathrm{M}$ for each marathon. Apart from the Boston marathon data, all other three marathons show fairly unimodal shapes. This can be accounted by the strict qualifying times and the higher number of available spots in these marathons, allowing non-qualifiers to dominate these marathons in terms of headcount. In contrast, the density of Boston marathon finishing time shows clearer bimodality, because the Boston marathon has a relatively laxed qualifying times and it has the least number of spots, giving more comparable numbers of runners in both sub-groups.

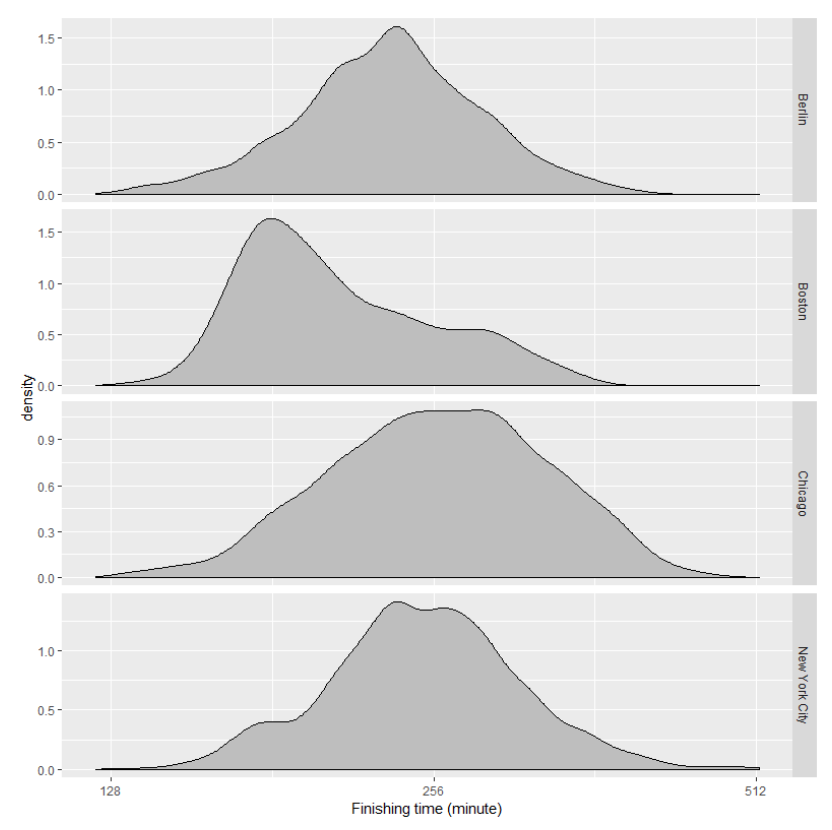

Figure 1: Kernel density plots for finishing times in $\log _{2}$ scale for male runners aged from 25 to 29.

To explore how runners' velocity changes from time to time, we let $v_{5 n}=\frac{5000}{t_{5 n}-t_{5 n-5}}$, the average velocity from interval $n-1$ to interval $n . g_{5 n}=\log \frac{v_{5 n+5}}{v_{5 n}}$, the log change of average velocity from interval $n$ to interval $n+1$, for $n=1, \ldots, 7$ and $t_{0}=0$.

Figure 2 plots $g_{5 n}$ against $t_{5 n}$ for $n=1, \ldots, 7$. It appears that faster runners attain more stable velocities throughout the marathon while slower runners have more uncertainties in their velocities. The distributions of $g_{5 n}$ for most runners who ran faster than $2 \mathrm{~m} / \mathrm{s}$ appear to be negatively skewed, i.e. more large slow downs are observed than large speed ups. In the later stages of the marathon, the number of runners having an average velocity of $1.5 \mathrm{~m} / \mathrm{s}$ or less increases, and among these slowest runners, large speed ups are frequently observed. A possible explanation of this phenomenon is that the slowest runners were recovering from walk breaks or injuries, causing such large log increases in velocity. Moreover, the times when runners slow down appear to be an arbitrary decision. For example, more slow downs are observed after the 20 kilometers check point. This suggests that many runners decide to slow down after a certain milestone has been reached. 


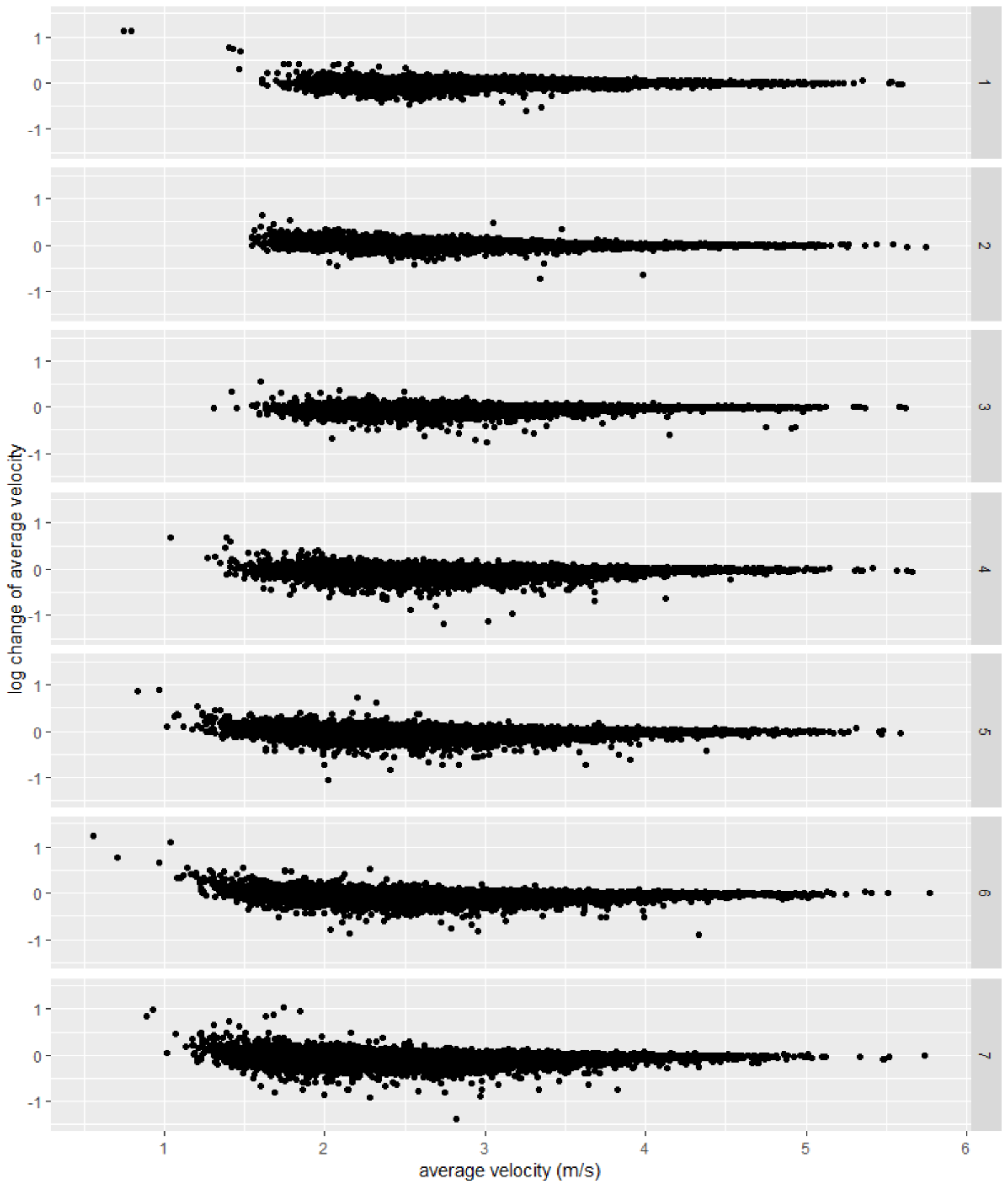

Figure 2: Log change of average velocity against average velocity at intervals 1 to 7 recorded in Chicago marathons from 2011 to 2018. Each plot contains 20000 randomly drawn samples from the datasets for clearer illustration. 


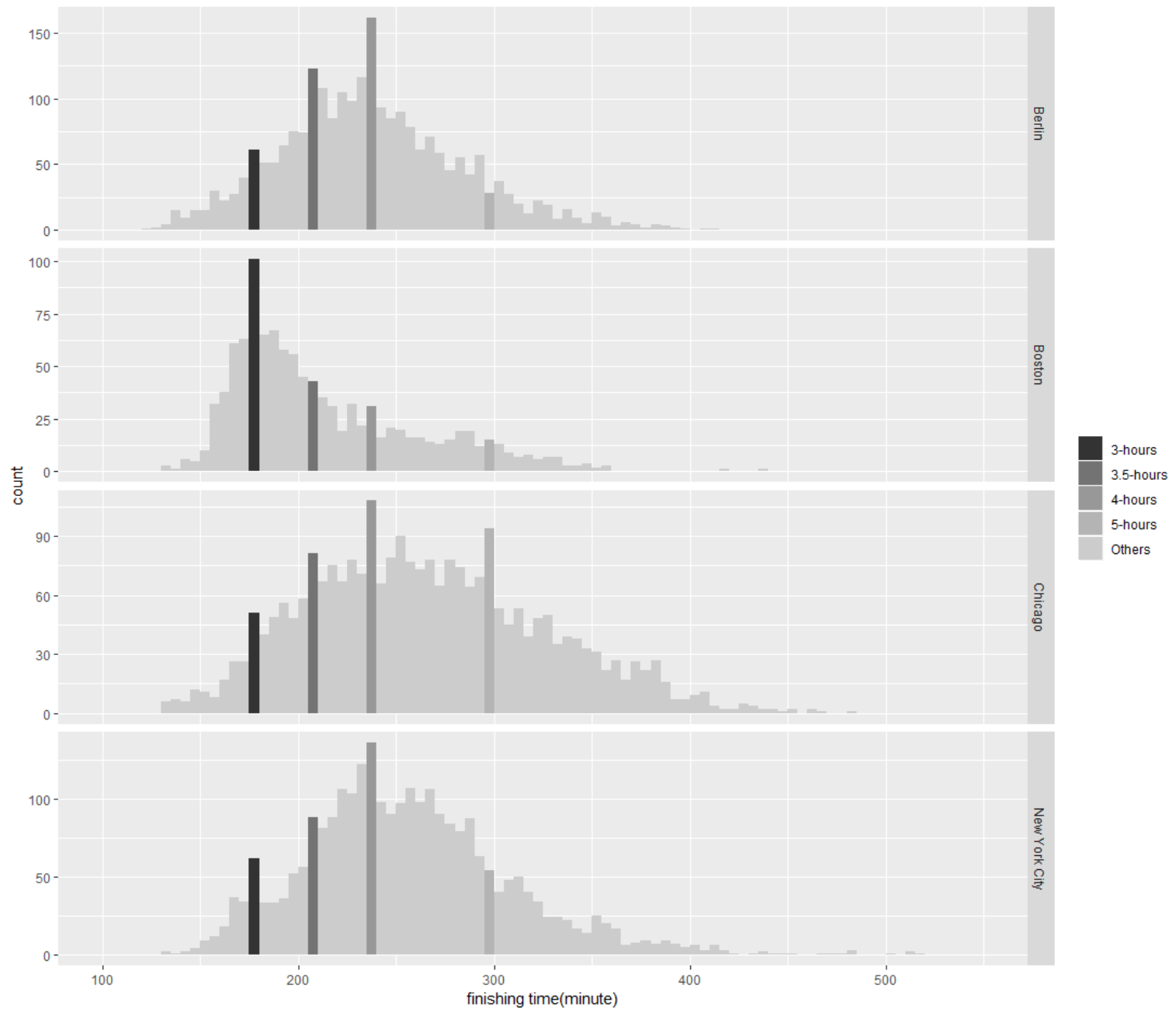

Figure 3: Histogram of finishing times with five minutes bin widths.

Another feature observed is about the heaped finishing and completion times. Histograms of finishing time ceiled to five minutes bins are plotted in Figure 3. We can see spikes at 3-hours marks, which are particularly clear in Boston marathon and New York city marathon. Similar spikes at 4-hour marks and 5-hour marks can be observed in some datasets. This suggests that there exists "heaped" psychological targets among runners, for example, capable runners adjust their velocity in order to achieve sub-3 hour or sub-4 hour results.

In later sections, we will propose a class of distributions to accommodate statistical properties observed in the interval completion times. We then develop a model to estimate change of average velocity.

\section{Interval completion time model}

The first part of the model describes a suitable class of distributions for finishing/interval completion times. As explained in Section 2, it is reasonable to believe that the general shape of finishing time 
distribution can be modelled by a mixture of two distributions, one for qualifiers and one for non-qualifiers.

Let $m_{f}$ be a quantity that estimates how fast a runner will finish the marathon. The distribution of $m_{f}$ for qualifiers can be assumed to have a similar shape as the fastest finishing time they submitted for marathon entry which is right truncated and negatively skewed. We let $m_{f}$ follow some unknown, bell shaped distribution for non-qualifiers. Since the finishing time is always positive, the actual finishing time can be described by the following process:

$$
t_{f}=m_{f} \exp \left(-g_{f}\right)
$$

where $-g_{f}$ follows some distribution describing the log deviation from the expected finishing time.

A similar process can describe how interval completion time changes from time to time:

$$
\begin{aligned}
& \left(t_{5 n+5}-t_{5 n}\right)=\left(t_{5 n}-t_{5 n-5}\right) \exp \left(-g_{5 n}\right), \\
& v_{5 n+5}=v_{5 n} \exp \left(g_{5 n}\right),
\end{aligned}
$$

where $g_{5 n}$ follows some distribution describing the log change of average velocity.

Even if we know the exact distributions of $\log t_{5 n}$ and $g_{5 n}$, the distribution of $\log t_{5 n}-g_{5 n}$ is very unlikely to have a closed form expression. This can be very inconvenient in practice. An appropriate alternative is to find a class of distributions that is flexible enough to approximate shapes of $t_{5 n}$. From figure 2, we can see that the relationship between $t_{5 n}$ and $g_{5 n}$ is rather complex, which would create changes in skewness and kurtosis, and thus changes the shape of the distribution of $t_{5 n+5}$. Without assuming the distribution of $g_{5 n}$, we consider a closed-form flexible distribution with a variety of shapes to approximate the distribution of $t_{5 n}$. We propose to use a mixture of skew exponential power (SEP) distributions (Zhu and Zinde-Walsh, 2009) to model interval completion times. Next, the distribution will be fitted to the datasets and comparisons will be carried out with the mixture of skew normal distributions, mixture of Gaussian distributions and other non mixture distributions.

\subsection{Data illustration}

Six classes of distributions were fitted to the four datasets: a) mixture of two skew exponential power distributions (2-SEP); b) mixture of two skew normal distributions (2-SN); c) mixture of two Gaussian distributions (2-N); d) Gaussian distribution (N); e) skew normal distribution (SN); f) skew exponential power distribution (SEP). The probability density function of a finite mixture of $K$ distributions has the form:

$$
f(x)=\sum_{k=1}^{K} \omega_{k} f_{k}(x),
$$

where $f_{k}(x)$ denotes the density of the $k$ th component distribution, $\omega_{1}+\cdots+\omega_{K}=1$ and $\omega_{k}>0$ for all $k \in 1, \ldots, K$.

The probability density function of the SEP distribution is as follows:

$$
f_{k}(x ; \mu, \sigma, \gamma, \xi)= \begin{cases}\frac{1}{\sigma} K_{E P}(\xi) \exp \left(-\frac{1}{\xi}\left|\frac{x-\mu}{2 \gamma \sigma}\right|^{\xi}\right), & \text { if } x \leq \mu, \\ \frac{1}{\sigma} K_{E P}(\xi) \exp \left(-\frac{1}{\xi}\left|\frac{x-\mu}{2(1-\gamma) \sigma}\right|^{\xi}\right), & \text { if } x>\mu\end{cases}
$$


for $-\infty<x<\infty,-\infty<\mu<\infty, \sigma>0,0<\gamma<1$ and $\xi>0$, where $K_{E P}(\rho)=\frac{1}{2 \rho^{1 / \xi} \Gamma(1+1 / \xi)}$.

The probability density function of the skew normal distribution is as follows:

$$
f_{k}(x ; \mu, \sigma, \alpha)=2 \phi\left(\frac{x-\mu}{\sigma}\right) \Phi\left(\alpha \frac{x-\mu}{\sigma}\right)
$$

for $-\infty<x<\infty,-\infty<\mu<\infty, \sigma>0$ and $-\infty<\alpha<\infty$, where $\phi$ and $\Phi$ denote, respectively, the probability density and cumulative distribution functions of a standard normal random variable.

The probability density function of the Gaussian distribution is as follows:

$$
f_{k}(x ; \mu, \sigma)=\frac{1}{\sqrt{2 \pi \sigma^{2}}} \exp \left(-\frac{(x-\mu)^{2}}{2 \sigma^{2}}\right)
$$

for $-\infty<x<\infty,-\infty<\mu<\infty$ and $\sigma>0$.

Maximum likelihood estimators of parameters were obtained via the EM algorithm (Demspter et al., 1977). Since bimodalities in most cases are insignificant, the parameters estimates obtained may not draw correct inference towards each subpopulation if initial parameters were not carefully specified. To circumvent this problem, we separated each dataset into two sets naively, one set with runners who finished faster than the qualifying time and the other set with runners who did not. Each set was fitted to the corresponding component distribution and the resulting estimates were set as the initial parameters for the EM algorithm.

Models were compared using Akaike information criteria (AIC) (Akaike, 1974). Since the number of qualifiers is very small compared to non-qualifiers in most datasets (except for the Boston marathon), the likelihood after adding a component distribution often would not increase dramatically. In view of this problem, we consider AIC to be more sensible to compare models than other criteria such as Bayesian information criteria (BIC) (Schwarz, 1978), because there is a good chance that the penalised likelihood function of BIC will over penalise the increase in the number of parameters. 


\begin{tabular}{|c|c|c|c|c|c|c|c|c|c|c|c|c|c|c|}
\hline Interval & Dist. & $\omega_{1}$ & $\mu_{1}$ & $\sigma_{1}$ & $\gamma_{1}$ & $\xi_{1}$ & $\omega_{2}$ & $\mu_{2}$ & $\sigma_{2}$ & $\gamma_{2}$ & $\xi_{2}$ & Log likelihood & $\mathrm{AIC}$ & $\mathrm{BIC}$ \\
\hline \multirow{6}{*}{1} & $2-\mathrm{N}$ & 0.05 & 7.07 & 0.06 & 0.95 & 7.41 & 0.16 & - & - & - & - & 925 & -1839 & -1810 \\
\hline & 2-SEP* & 0.10 & 7.12 & 0.61 & 0.07 & 2.01 & 0.90 & 7.38 & 0.39 & 0.13 & 1.67 & 949 & -1880 & -1827 \\
\hline & $2-\mathrm{SN}^{* *}$ & 0.11 & 7.09 & 0.08 & 0.06 & 0.89 & 7.28 & 0.20 & 2.10 & - & - & 947 & -1879 & -1838 \\
\hline & $\mathrm{N}$ & - & 7.39 & 0.17 & - & - & - & - & - & - & - & 909 & -1814 & -1802 \\
\hline & SEP & - & 7.40 & 0.52 & 0.16 & 1.58 & - & - & - & - & - & 926 & -1844 & -1820 \\
\hline & $\mathrm{SN}$ & - & 7.39 & 0.17 & -0.01 & - & - & - & - & - & - & 909 & -1812 & -1794 \\
\hline \multirow{6}{*}{2} & $2-\mathrm{N}$ & 0.03 & 7.07 & 0.05 & 0.97 & 7.39 & 0.17 & - & - & - & - & 857 & -1704 & -1675 \\
\hline & 2-SEP & 0.09 & 7.15 & 0.82 & 0.06 & 1.94 & 0.91 & 7.36 & 0.38 & 0.14 & 1.62 & 887 & -1757 & -1704 \\
\hline & $2-\mathrm{SN}^{* * *}$ & 0.10 & 7.17 & 0.11 & -5.45 & 0.90 & 7.26 & 0.22 & 2.44 & - & - & 886 & -1758 & -1717 \\
\hline & $\mathrm{N}$ & - & 7.38 & 0.17 & - & - & - & - & - & - & - & 851 & -1698 & -1686 \\
\hline & SEP & - & 7.38 & 0.50 & 0.16 & 1.55 & - & - & - & - & - & 870 & -1732 & -1708 \\
\hline & $\mathrm{SN}$ & - & 7.27 & 0.21 & 0.92 & - & - & - & - & - & - & 855 & -1705 & -1687 \\
\hline \multirow{6}{*}{3} & $2-\mathrm{N}$ & 0.03 & 7.08 & 0.05 & 0.97 & 7.41 & 0.17 & - & - & - & - & 769 & -1527 & -1498 \\
\hline & $2-\mathrm{SEP}^{*}$ & 0.06 & 7.16 & 0.99 & 0.05 & 2.03 & 0.94 & 7.40 & 0.45 & 0.15 & 1.47 & 809 & -1599 & -1546 \\
\hline & $2-\mathrm{SN}^{* *}$ & 0.10 & 7.17 & 0.11 & -4.86 & 0.90 & 7.27 & 0.23 & 2.48 & - & - & 801 & -1589 & -1548 \\
\hline & $\mathrm{N}$ & - & 7.40 & 0.18 & - & - & - & - & - & - & - & 763 & -1521 & -1510 \\
\hline & SEP & - & 7.40 & 0.50 & 0.16 & 1.52 & - & - & - & - & - & 785 & -1561 & -1538 \\
\hline & $\mathrm{SN}$ & - & 7.28 & 0.22 & 0.98 & - & - & - & - & - & - & 768 & -1530 & -1513 \\
\hline \multirow{6}{*}{4} & $2-\mathrm{N}$ & 0.02 & 7.09 & 0.05 & 0.98 & 7.44 & 0.19 & - & - & - & - & 503 & -997 & -967 \\
\hline & 2-SEP & 0.10 & 7.15 & 0.80 & 0.06 & 1.76 & 0.90 & 7.36 & 0.29 & 0.15 & 1.71 & 548 & -1079 & -1026 \\
\hline & $2-\mathrm{SN}^{* * *}$ & 0.10 & 7.18 & 0.11 & -6.04 & 0.90 & 7.27 & 0.26 & 3.14 & - & - & 549 & -1083 & -1042 \\
\hline & $\mathrm{N}$ & - & 7.43 & 0.20 & - & - & - & - & - & - & - & 499 & -993 & -982 \\
\hline & SEP & - & 7.41 & 0.47 & 0.18 & 1.52 & - & - & - & - & - & 527 & -1045 & -1022 \\
\hline & $\mathrm{SN}$ & - & 7.27 & 0.26 & 1.27 & - & - & - & - & - & - & 514 & -1021 & -1003 \\
\hline \multirow{6}{*}{5} & $2-\mathrm{N}$ & 0.02 & 7.12 & 0.04 & 0.98 & 7.51 & 0.22 & - & - & - & - & 188 & -367 & -337 \\
\hline & 2-SEP & 0.09 & 7.16 & 0.68 & 0.06 & 1.53 & 0.91 & 7.40 & 0.27 & 0.18 & 1.89 & 227 & -437 & -384 \\
\hline & $2-\mathrm{SN}^{* * *} *$ & 0.10 & 7.20 & 0.11 & -3.10 & 0.90 & 7.30 & 0.31 & 3.67 & - & - & 228 & -441 & -400 \\
\hline & $\mathrm{N}$ & - & 7.50 & 0.23 & - & - & - & - & - & - & - & 182 & -361 & -349 \\
\hline & SEP & - & 7.44 & 0.42 & 0.21 & 1.74 & - & - & - & - & - & 205 & -401 & -378 \\
\hline & SN & - & 7.29 & 0.30 & 1.52 & - & - & - & - & - & - & 203 & -400 & -382 \\
\hline \multirow{6}{*}{6} & $2-\mathrm{N}$ & 0.02 & 7.10 & 0.04 & 0.98 & 7.51 & 0.23 & - & - & - & - & 30 & -50 & -21 \\
\hline & $2-\mathrm{SEP}^{*}$ & 0.10 & 7.14 & 0.68 & 0.06 & 1.53 & 0.90 & 7.37 & 0.23 & 0.21 & 2.28 & 58 & -97 & -45 \\
\hline & $2-\mathrm{SN}^{* *}$ & 0.08 & 7.17 & 0.11 & -4.06 & 0.92 & 7.29 & 0.33 & 3.02 & - & - & 54 & -94 & -53 \\
\hline & $\mathrm{N}$ & - & 7.50 & 0.24 & - & - & - & - & - & - & - & 25 & -46 & -34 \\
\hline & SEP & - & 7.45 & 0.43 & 0.24 & 1.97 & - & - & - & - & - & 39 & -70 & -47 \\
\hline & $\mathrm{SN}$ & - & 7.51 & 0.24 & 0.00 & - & - & - & - & - & - & 25 & -44 & -26 \\
\hline \multirow{6}{*}{7} & $2-\mathrm{N}$ & 0.05 & 7.20 & 0.11 & 0.95 & 7.59 & 0.23 & - & - & - & - & -39 & 88 & 117 \\
\hline & 2-SEP* & 0.10 & 7.16 & 0.51 & 0.08 & 1.18 & 0.90 & 7.48 & 0.30 & 0.23 & 2.96 & -20 & 58 & 111 \\
\hline & 2-SN & 0.06 & 7.21 & 0.12 & -0.07 & 0.94 & 7.48 & 0.26 & 0.66 & - & - & -39 & 92 & 133 \\
\hline & $\mathrm{N}$ & - & 7.57 & 0.25 & - & - & - & - & - & - & - & -42 & 89 & 101 \\
\hline & $\mathrm{SEP}^{* *}$ & - & 7.57 & 0.49 & 0.26 & 2.44 & - & - & - & - & - & -34 & 75 & 99 \\
\hline & SN & - & 7.58 & 0.25 & -0.01 & - & - & - & - & - & - & -42 & 91 & 109 \\
\hline \multirow{6}{*}{8} & $2-\mathrm{N}$ & 0.07 & 7.25 & 0.14 & 0.93 & 7.63 & 0.22 & - & - & - & - & 73 & -137 & -108 \\
\hline & 2-SEP & 0.10 & 7.21 & 0.50 & 0.11 & 1.83 & 0.90 & 7.56 & 0.37 & 0.22 & 2.56 & 76 & -135 & -82 \\
\hline & 2-SN & 0.08 & 7.25 & 0.14 & -0.01 & 0.92 & 7.54 & 0.24 & 0.58 & - & - & 73 & -132 & -91 \\
\hline & $\mathrm{N}^{* *}$ & - & 7.60 & 0.24 & - & - & - & - & - & - & - & 70 & -135 & -123 \\
\hline & SEP* & - & 7.62 & 0.52 & 0.24 & 2.20 & - & - & - & - & - & 73 & -138 & -114 \\
\hline & $\mathrm{SN}$ & - & 7.72 & 0.26 & -0.68 & - & - & - & - & - & - & 70 & -134 & -117 \\
\hline
\end{tabular}

Table 2: Fitted parameters for interval completion time distributions of New York City marathon 2017 (* denotes distributions with lowest AIC; ** denotes distributions with lowest BIC). 


\begin{tabular}{|c|c|c|c|c|c|c|c|c|c|c|c|c|c|c|}
\hline Interval & Dist. & $\omega_{1}$ & $\mu_{1}$ & $\sigma_{1}$ & $\gamma_{1}$ & $\xi_{1}$ & $\omega_{2}$ & $\mu_{2}$ & $\sigma_{2}$ & $\gamma_{2}$ & $\xi_{2}$ & Log likelihood & $\mathrm{AIC}$ & $\mathrm{BIC}$ \\
\hline \multirow{6}{*}{1} & $2-\mathrm{N}$ & 0.74 & 7.11 & 0.09 & 0.26 & 7.49 & 0.10 & - & - & - & - & 523 & -1035 & -1010 \\
\hline & 2-SEP* & 0.67 & 7.14 & 0.71 & 0.06 & 1.53 & 0.33 & 7.54 & 0.76 & 0.12 & 1.46 & 558 & -1098 & -1053 \\
\hline & $2-\mathrm{SN}^{* *}$ & 0.66 & 7.18 & 0.12 & -4.02 & 0.34 & 7.61 & 0.24 & -3.73 & - & - & 553 & -1092 & -1057 \\
\hline & $\mathrm{N}$ & - & 7.21 & 0.19 & - & - & - & - & - & - & - & 269 & -533 & -523 \\
\hline & SEP & - & 7.09 & 0.27 & 0.13 & 1.11 & - & - & - & - & - & 398 & -787 & -767 \\
\hline & $\mathrm{SN}$ & - & 6.99 & 0.29 & 4.27 & - & - & - & - & - & - & 381 & -756 & -741 \\
\hline \multirow{6}{*}{2} & $2-\mathrm{N}$ & 0.73 & 7.10 & 0.08 & 0.27 & 7.45 & 0.12 & - & - & - & - & 525 & -1040 & -1015 \\
\hline & 2-SEP* & 0.68 & 7.14 & 0.72 & 0.06 & 1.51 & 0.32 & 7.45 & 0.57 & 0.14 & 1.82 & 556 & -1095 & -1050 \\
\hline & $2-\mathrm{SN}^{* *}$ & 0.68 & 7.18 & 0.12 & -3.93 & 0.32 & 7.42 & 0.14 & -0.02 & - & - & 553 & -1092 & -1057 \\
\hline & $\mathrm{N}$ & - & 7.20 & 0.18 & - & - & - & - & - & - & - & 314 & -625 & -615 \\
\hline & SEP & - & 7.08 & 0.26 & 0.13 & 1.16 & - & - & - & - & - & 448 & -887 & -867 \\
\hline & $\mathrm{SN}$ & - & 7.20 & 0.18 & 0.00 & - & - & - & - & - & - & 314 & -623 & -608 \\
\hline \multirow{6}{*}{3} & $2-\mathrm{N}$ & 0.70 & 7.11 & 0.08 & 0.30 & 7.45 & 0.14 & - & - & - & - & 479 & -949 & -924 \\
\hline & 2-SEP*** & 0.69 & 7.14 & 0.60 & 0.07 & 1.51 & 0.31 & 7.43 & 0.48 & 0.13 & 1.50 & 496 & -974 & -929 \\
\hline & 2-SN & 0.73 & 7.12 & 0.09 & 0.02 & 0.27 & 7.34 & 0.18 & 3.16 & - & - & 485 & -956 & -921 \\
\hline & $\mathrm{N}$ & - & 7.21 & 0.18 & - & - & - & - & - & - & - & 294 & -584 & -574 \\
\hline & SEP & - & 7.09 & 0.25 & 0.13 & 1.19 & - & - & - & - & - & 427 & -847 & -827 \\
\hline & $\mathrm{SN}$ & - & 7.21 & 0.18 & 0.00 & - & - & - & - & - & - & 294 & -582 & -567 \\
\hline \multirow{6}{*}{4} & $2-\mathrm{N}^{* *}$ & 0.60 & 7.13 & 0.08 & 0.40 & 7.44 & 0.20 & - & - & - & - & 335 & -661 & -636 \\
\hline & 2-SEP* & 0.69 & 7.14 & 0.49 & 0.08 & 1.42 & 0.31 & 7.43 & 0.33 & 0.15 & 1.98 & 341 & -663 & -618 \\
\hline & 2-SN & 0.68 & 7.13 & 0.09 & 0.05 & 0.32 & 7.32 & 0.24 & 2.66 & - & - & 335 & -656 & -621 \\
\hline & $\mathrm{N}$ & - & 7.25 & 0.21 & - & - & - & - & - & - & - & 159 & -315 & -305 \\
\hline & SEP & - & 7.09 & 0.22 & 0.15 & 1.26 & - & - & - & - & - & 303 & -598 & -578 \\
\hline & $\mathrm{SN}$ & - & 7.00 & 0.32 & 5.01 & - & - & - & - & - & - & 289 & -572 & -557 \\
\hline \multirow{6}{*}{5} & $2-\mathrm{N}^{* * *}$ & 0.58 & 7.14 & 0.09 & 0.42 & 7.48 & 0.22 & - & - & - & - & 237 & -464 & -439 \\
\hline & 2-SEP & 0.70 & 7.13 & 0.41 & 0.09 & 1.53 & 0.30 & 7.47 & 0.30 & 0.17 & 2.11 & 240 & -463 & -418 \\
\hline & $2-\mathrm{SN}$ & 0.70 & 7.07 & 0.13 & 1.34 & 0.30 & 7.38 & 0.26 & 3.11 & - & - & 235 & -456 & -421 \\
\hline & $\mathrm{N}$ & - & 7.28 & 0.23 & - & - & - & - & - & - & - & 54 & -104 & -94 \\
\hline & SEP & - & 7.09 & 0.19 & 0.17 & 1.26 & - & - & - & - & - & 211 & -413 & -393 \\
\hline & SN & - & 7.01 & 0.36 & 5.71 & - & - & - & - & - & - & 195 & -383 & -368 \\
\hline \multirow{6}{*}{6} & $2-\mathrm{N}^{* *}$ & 0.53 & 7.17 & 0.10 & 0.47 & 7.52 & 0.21 & - & - & - & - & 151 & -292 & -267 \\
\hline & $2-\mathrm{SEP}^{*}$ & 0.69 & 7.15 & 0.36 & 0.11 & 1.52 & 0.31 & 7.49 & 0.24 & 0.18 & 2.94 & 157 & -296 & -251 \\
\hline & 2-SN & 0.68 & 7.08 & 0.18 & 2.05 & 0.32 & 7.49 & 0.21 & 1.01 & - & - & 150 & -286 & -251 \\
\hline & $\mathrm{N}$ & - & 7.34 & 0.24 & - & - & - & - & - & - & - & 18 & -33 & -23 \\
\hline & SEP & - & 7.13 & 0.19 & 0.20 & 1.60 & - & - & - & - & - & 130 & -253 & -233 \\
\hline & $\mathrm{SN}$ & - & 7.04 & 0.38 & 5.48 & - & - & - & - & - & - & 126 & -247 & -232 \\
\hline \multirow{6}{*}{7} & $2-\mathrm{N}^{* *}$ & 0.50 & 7.21 & 0.11 & 0.50 & 7.59 & 0.21 & - & - & - & - & 42 & -75 & -50 \\
\hline & 2-SEP* & 0.69 & 7.18 & 0.32 & 0.14 & 1.60 & 0.31 & 7.57 & 0.28 & 0.19 & 3.25 & 49 & -79 & -34 \\
\hline & 2-SN & 0.70 & 7.09 & 0.24 & 2.60 & 0.30 & 7.68 & 0.18 & 0.09 & - & - & 43 & -73 & -38 \\
\hline & $\mathrm{N}$ & - & 7.40 & 0.25 & - & - & - & - & - & - & - & -49 & 101 & 111 \\
\hline & SEP & - & 7.19 & 0.22 & 0.24 & 2.18 & - & - & - & - & - & 28 & -49 & -29 \\
\hline & $\mathrm{SN}$ & - & 7.40 & 0.25 & 0.00 & - & - & - & - & - & - & -49 & 103 & 118 \\
\hline \multirow{6}{*}{8} & $2-\mathrm{N}^{* *}$ & 0.35 & 7.19 & 0.11 & 0.65 & 7.55 & 0.23 & - & - & - & - & -12 & 34 & 59 \\
\hline & 2-SEP* & 0.68 & 7.18 & 0.28 & 0.17 & 1.59 & 0.32 & 7.56 & 0.29 & 0.19 & 2.76 & -8 & 34 & 79 \\
\hline & 2-SN & 0.70 & 7.08 & 0.29 & 2.90 & 0.30 & 7.54 & 0.23 & 1.30 & - & - & -14 & 41 & 76 \\
\hline & $\mathrm{N}$ & - & 7.42 & 0.26 & - & - & - & - & - & - & - & -65 & 133 & 143 \\
\hline & SEP & - & 7.25 & 0.29 & 0.27 & 2.74 & - & - & - & - & - & -17 & 41 & 61 \\
\hline & $\mathrm{SN}$ & - & 7.42 & 0.26 & 0.00 & - & - & - & - & - & - & -65 & 135 & 150 \\
\hline
\end{tabular}

Table 3: Fitted parameters for interval completion time distributions of Boston marathon 2017 (* denotes distributions with lowest $\mathrm{AIC}$; ${ }^{* *}$ denotes distributions with lowest $\mathrm{BIC}$ ). 


\begin{tabular}{|c|c|c|c|c|c|c|c|c|c|c|c|c|c|c|}
\hline Interval & Dist. & $\omega_{1}$ & $\mu_{1}$ & $\sigma_{1}$ & $\gamma_{1}$ & $\xi_{1}$ & $\omega_{2}$ & $\mu_{2}$ & $\sigma_{2}$ & $\gamma_{2}$ & $\xi_{2}$ & Log likelihood & $\mathrm{AIC}$ & $\mathrm{BIC}$ \\
\hline \multirow{6}{*}{1} & 2-SEP* & 0.08 & 7.16 & 0.98 & 0.10 & 3.75 & 0.92 & 7.37 & 0.47 & 0.16 & 2.53 & 806 & -1594 & -1542 \\
\hline & $2-\mathrm{N}$ & 0.07 & 7.03 & 0.12 & 0.93 & 7.38 & 0.15 & - & - & - & - & 794 & -1578 & -1549 \\
\hline & 2-SN & 0.08 & 7.18 & 0.17 & -19.63 & 0.92 & 7.39 & 0.15 & 0.02 & - & - & 799 & -1584 & -1544 \\
\hline & $\mathrm{N}$ & - & 7.36 & 0.17 & - & - & - & - & - & - & - & 767 & -1530 & -1519 \\
\hline & SEP & - & 7.42 & 0.61 & 0.17 & 1.98 & - & - & - & - & - & 794 & -1580 & -1557 \\
\hline & $\mathrm{SN}^{*}$ & - & 7.53 & 0.24 & -1.78 & - & - & - & - & - & - & 795 & -1585 & -1567 \\
\hline \multirow{6}{*}{2} & 2-SEP & 0.08 & 7.16 & 0.99 & 0.10 & 3.73 & 0.92 & 7.34 & 0.40 & 0.16 & 2.12 & 683 & -1348 & -1296 \\
\hline & $2-\mathrm{N}$ & 0.02 & 6.93 & 0.07 & 0.98 & 7.37 & 0.17 & - & - & - & - & 675 & -1339 & -1311 \\
\hline & $2-\mathrm{SN}^{*}$ & 0.10 & 7.16 & 0.16 & -36.15 & 0.90 & 7.25 & 0.21 & 1.66 & - & - & 681 & -1349 & -1309 \\
\hline & $\mathrm{N}$ & - & 7.36 & 0.18 & - & - & - & - & - & - & - & 663 & -1322 & -1311 \\
\hline & SEP & - & 7.38 & 0.54 & 0.17 & 1.79 & - & - & - & - & - & 670 & -1332 & -1309 \\
\hline & $\mathrm{SN}^{* *}$ & - & 7.49 & 0.22 & -1.05 & - & - & - & - & - & - & 668 & -1330 & -1312 \\
\hline \multirow{6}{*}{3} & 2-SEP & 0.07 & 7.15 & 0.99 & 0.11 & 4.29 & 0.93 & 7.35 & 0.43 & 0.15 & 1.90 & 713 & -1408 & -1356 \\
\hline & $2-\mathrm{N}$ & 0.02 & 6.94 & 0.07 & 0.98 & 7.37 & 0.17 & - & - & - & - & 707 & -1404 & -1375 \\
\hline & $2-\mathrm{SN}^{* * *}$ & 0.11 & 7.17 & 0.15 & -29.06 & 0.89 & 7.25 & 0.21 & 1.89 & - & - & 719 & -1423 & -1383 \\
\hline & $\mathrm{N}$ & - & 7.36 & 0.18 & - & - & - & - & - & - & - & 694 & -1384 & -1372 \\
\hline & SEP & - & 7.38 & 0.54 & 0.17 & 1.66 & - & - & - & - & - & 705 & -1403 & -1380 \\
\hline & $\mathrm{SN}$ & - & 7.49 & 0.22 & -1.05 & - & - & - & - & - & - & 699 & -1391 & -1374 \\
\hline \multirow{6}{*}{4} & 2-SEP* & 0.05 & 7.15 & 0.99 & 0.11 & 5.52 & 0.95 & 7.36 & 0.46 & 0.16 & 1.65 & 581 & -1145 & -1093 \\
\hline & $2-\mathrm{N}$ & 0.02 & 6.94 & 0.07 & 0.98 & 7.38 & 0.18 & - & - & - & - & 568 & -1127 & -1098 \\
\hline & $2-\mathrm{SN}$ & 0.05 & 7.13 & 0.16 & -63.71 & 0.95 & 7.24 & 0.23 & 1.56 & - & - & 567 & -1119 & -1079 \\
\hline & $\mathrm{N}$ & - & 7.37 & 0.19 & - & - & - & - & - & - & - & 562 & -1120 & -1109 \\
\hline & $\mathrm{SEP}^{* *}$ & - & 7.38 & 0.52 & 0.17 & 1.57 & - & - & - & - & - & 576 & -1145 & -1122 \\
\hline & $\mathrm{SN}$ & - & 7.42 & 0.20 & -0.35 & - & - & - & - & - & - & 562 & -1118 & -1101 \\
\hline \multirow{6}{*}{5} & 2-SEP & 0.07 & 7.16 & 0.99 & 0.09 & 3.12 & 0.93 & 7.35 & 0.36 & 0.17 & 1.70 & 375 & -732 & -680 \\
\hline & $2-\mathrm{N}$ & 0.01 & 7.06 & 0.14 & 0.99 & 7.40 & 0.21 & - & - & - & - & 346 & -683 & -654 \\
\hline & $2-\mathrm{SN}^{* * *}$ & 0.10 & 7.17 & 0.15 & -51.50 & 0.90 & 7.24 & 0.27 & 2.87 & - & - & 380 & -746 & -706 \\
\hline & $\mathrm{N}$ & - & 7.40 & 0.21 & - & - & - & - & - & - & - & 347 & -691 & -679 \\
\hline & SEP & - & 7.38 & 0.47 & 0.19 & 1.55 & - & - & - & - & - & 367 & -726 & -703 \\
\hline & $\mathrm{SN}$ & - & 7.25 & 0.26 & 1.09 & - & - & - & - & - & - & 354 & -702 & -685 \\
\hline \multirow{6}{*}{6} & 2-SEP & 0.08 & 7.16 & 0.99 & 0.09 & 2.78 & 0.92 & 7.37 & 0.33 & 0.19 & 1.79 & 151 & -284 & -232 \\
\hline & $2-\mathrm{N}$ & 0.01 & 7.20 & 0.16 & 0.99 & 7.44 & 0.23 & - & - & - & - & 123 & -235 & -207 \\
\hline & $2-\mathrm{SN}^{*}$ & 0.09 & 7.16 & 0.15 & -17.24 & 0.91 & 7.25 & 0.31 & 2.95 & - & - & 152 & -289 & -249 \\
\hline & $\mathrm{N}$ & - & 7.44 & 0.23 & - & - & - & - & - & - & - & 123 & -242 & -230 \\
\hline & $\mathrm{SEP}^{* *}$ & - & 7.40 & 0.45 & 0.21 & 1.62 & - & - & - & - & - & 141 & -273 & -250 \\
\hline & $\mathrm{SN}$ & - & 7.25 & 0.29 & 1.26 & - & - & - & - & - & - & 133 & -260 & -243 \\
\hline \multirow{6}{*}{7} & 2-SEP & 0.08 & 7.14 & 0.85 & 0.09 & 2.48 & 0.92 & 7.38 & 0.32 & 0.20 & 2.07 & 138 & -257 & -205 \\
\hline & $2-\mathrm{N}$ & 0.02 & 7.11 & 0.13 & 0.98 & 7.46 & 0.23 & - & - & - & - & 122 & -233 & -204 \\
\hline & $2-\mathrm{SN}^{*}$ & 0.09 & 7.17 & 0.15 & -7.94 & 0.91 & 7.26 & 0.31 & 2.83 & - & - & 136 & -258 & -218 \\
\hline & $\mathrm{N}^{* *}$ & - & 7.45 & 0.23 & - & - & - & - & - & - & - & 122 & -241 & -229 \\
\hline & SEP & - & 7.42 & 0.46 & 0.22 & 1.81 & - & - & - & - & - & 129 & -249 & -226 \\
\hline & $\mathrm{SN}$ & - & 7.30 & 0.28 & 1.02 & - & - & - & - & - & - & 126 & -246 & -228 \\
\hline \multirow{6}{*}{8} & $2-\mathrm{SEP}^{*}$ & 0.08 & 7.14 & 0.66 & 0.10 & 2.51 & 0.92 & 7.43 & 0.34 & 0.21 & 2.15 & 62 & -107 & -55 \\
\hline & $2-\mathrm{N}$ & 0.02 & 7.19 & 0.15 & 0.98 & 7.51 & 0.23 & - & - & - & - & 53 & -96 & -67 \\
\hline & $2-\mathrm{SN}$ & 0.08 & 7.20 & 0.15 & -2.53 & 0.92 & 7.31 & 0.31 & 2.39 & - & - & 57 & -101 & -60 \\
\hline & $\mathrm{N}^{* *}$ & - & 7.50 & 0.24 & - & - & - & - & - & - & - & 53 & -102 & -91 \\
\hline & SEP & - & 7.47 & 0.46 & 0.23 & 1.94 & - & - & - & - & - & 57 & -106 & -83 \\
\hline & $\mathrm{SN}$ & - & 7.50 & 0.24 & 0.00 & - & - & - & - & - & - & 53 & -100 & -83 \\
\hline
\end{tabular}

Table 4: Fitted parameters for interval completion time distributions of Berlin marathon 2017 (* denotes distributions with lowest $\mathrm{AIC}$; ${ }^{* *}$ denotes distributions with lowest $\mathrm{BIC}$ ). 


\begin{tabular}{|c|c|c|c|c|c|c|c|c|c|c|c|c|c|c|}
\hline Interval & Dist. & $\omega_{1}$ & $\mu_{1}$ & $\sigma_{1}$ & $\gamma_{1}$ & $\xi_{1}$ & $\omega_{2}$ & $\mu_{2}$ & $\sigma_{2}$ & $\gamma_{2}$ & $\xi_{2}$ & Log likelihood & $\mathrm{AIC}$ & $\mathrm{BIC}$ \\
\hline \multirow{6}{*}{1} & $2-\mathrm{N}$ & 0.08 & 7.10 & 0.13 & 0.92 & 7.42 & 0.17 & - & - & - & - & 712 & -1415 & -1386 \\
\hline & $2-\mathrm{SEP}^{*}$ & 0.09 & 7.17 & 0.97 & 0.06 & 1.14 & 0.91 & 7.42 & 0.47 & 0.16 & 2.23 & 730 & -1442 & -1389 \\
\hline & 2-SN & 0.08 & 7.18 & 0.14 & -30.29 & 0.92 & 7.40 & 0.16 & 0.20 & - & - & 723 & -1432 & -1391 \\
\hline & $\mathrm{N}$ & - & 7.40 & 0.18 & - & - & - & - & - & - & - & 700 & -1395 & -1384 \\
\hline & $\mathrm{SEP}^{* *}$ & - & 7.45 & 0.58 & 0.18 & 2.02 & - & - & - & - & - & 715 & -1422 & -1398 \\
\hline & $\mathrm{SN}$ & - & 7.40 & 0.18 & 0.00 & - & - & - & - & - & - & 700 & -1393 & -1376 \\
\hline \multirow{6}{*}{2} & $2-\mathrm{N}$ & 0.04 & 7.16 & 0.14 & 0.96 & 7.42 & 0.19 & - & - & - & - & 506 & -1002 & -973 \\
\hline & 2-SEP* & 0.09 & 7.16 & 0.94 & 0.05 & 0.99 & 0.91 & 7.38 & 0.38 & 0.17 & 2.01 & 524 & -1029 & -977 \\
\hline & 2-SN & 0.08 & 7.17 & 0.14 & -24.48 & 0.92 & 7.27 & 0.25 & 1.76 & - & - & 517 & -1019 & -978 \\
\hline & $\mathrm{N}^{* *}$ & - & 7.41 & 0.20 & - & - & - & - & - & - & - & 507 & -1010 & -998 \\
\hline & SEP & - & 7.40 & 0.49 & 0.19 & 1.88 & - & - & - & - & - & 508 & -1008 & -985 \\
\hline & $\mathrm{SN}$ & - & 7.41 & 0.20 & -0.01 & - & - & - & - & - & - & 507 & -1008 & -990 \\
\hline \multirow{6}{*}{3} & $2-\mathrm{N}$ & 0.04 & 7.18 & 0.14 & 0.96 & 7.42 & 0.19 & - & - & - & - & 516 & -1021 & -992 \\
\hline & 2-SEP* & 0.08 & 7.15 & 0.90 & 0.05 & 0.82 & 0.92 & 7.37 & 0.37 & 0.18 & 2.15 & 527 & -1035 & -983 \\
\hline & 2-SN & 0.07 & 7.18 & 0.15 & -17.93 & 0.93 & 7.27 & 0.24 & 1.65 & - & - & 522 & -1030 & -989 \\
\hline & $\mathrm{N}^{* *}$ & - & 7.41 & 0.20 & - & - & - & - & - & - & - & 516 & -1028 & -1017 \\
\hline & SEP & - & 7.40 & 0.49 & 0.19 & 1.92 & - & - & - & - & - & 517 & -1026 & -1003 \\
\hline & $\mathrm{SN}$ & - & 7.41 & 0.20 & -0.01 & - & - & - & - & - & - & 516 & -1026 & -1009 \\
\hline \multirow{6}{*}{4} & $2-\mathrm{N}$ & 0.04 & 7.20 & 0.09 & 0.96 & 7.46 & 0.22 & - & - & - & - & 197 & -383 & -354 \\
\hline & 2-SEP* & 0.08 & 7.16 & 0.99 & 0.05 & 1.05 & 0.92 & 7.36 & 0.31 & 0.21 & 2.31 & 209 & -399 & -347 \\
\hline & 2-SN & 0.06 & 7.17 & 0.15 & -28.53 & 0.94 & 7.26 & 0.30 & 2.00 & - & - & 202 & -390 & -349 \\
\hline & $\mathrm{N}$ & - & 7.45 & 0.22 & - & - & - & - & - & - & - & 193 & -383 & -371 \\
\hline & SEP & - & 7.41 & 0.45 & 0.22 & 1.99 & - & - & - & - & - & 200 & -393 & -369 \\
\hline & $\mathrm{SN}^{* *}$ & - & 7.28 & 0.28 & 1.16 & - & - & - & - & - & - & 200 & -394 & -376 \\
\hline \multirow{6}{*}{5} & $2-\mathrm{N}$ & 0.17 & 7.30 & 0.13 & 0.83 & 7.53 & 0.26 & - & - & - & - & -128 & 267 & 296 \\
\hline & $2-\mathrm{SEP}^{*}$ & 0.08 & 7.16 & 0.96 & 0.06 & 1.19 & 0.92 & 7.34 & 0.24 & 0.24 & 2.73 & -117 & 252 & 305 \\
\hline & 2-SN & 0.07 & 7.18 & 0.15 & -26.75 & 0.93 & 7.25 & 0.36 & 2.94 & - & - & -136 & 287 & 328 \\
\hline & $\mathrm{N}$ & - & 7.49 & 0.26 & - & - & - & - & - & - & - & -151 & 307 & 319 \\
\hline & $\mathrm{SEP}^{* *}$ & - & 7.42 & 0.41 & 0.27 & 2.28 & - & - & - & - & - & -129 & 266 & 289 \\
\hline & SN & - & 7.49 & 0.26 & 0.00 & - & - & - & - & - & - & -151 & 309 & 326 \\
\hline \multirow{6}{*}{6} & $2-\mathrm{N}$ & 0.14 & 7.27 & 0.13 & 0.86 & 7.59 & 0.26 & - & - & - & - & -299 & 607 & 636 \\
\hline & $2-\mathrm{SEP}^{*}$ & 0.09 & 7.16 & 0.83 & 0.06 & 0.89 & 0.91 & 7.41 & 0.27 & 0.29 & 3.82 & -276 & 570 & 623 \\
\hline & 2-SN & 0.02 & 7.17 & 0.03 & -1.33 & 0.98 & 7.48 & 0.28 & 0.38 & - & - & -306 & 625 & 666 \\
\hline & $\mathrm{N}$ & - & 7.55 & 0.27 & - & - & - & - & - & - & - & -319 & 642 & 653 \\
\hline & $\mathrm{SEP}^{* *}$ & - & 7.53 & 0.48 & 0.31 & 2.95 & - & - & - & - & - & -286 & 580 & 604 \\
\hline & SN & - & 7.55 & 0.27 & 0.00 & - & - & - & - & - & - & -319 & 644 & 661 \\
\hline \multirow{6}{*}{7} & $2-\mathrm{N}$ & 0.51 & 7.41 & 0.21 & 0.49 & 7.83 & 0.17 & - & - & - & - & -352 & 714 & 743 \\
\hline & 2-SEP & 0.09 & 7.19 & 0.78 & 0.08 & 1.58 & 0.91 & 7.60 & 0.42 & 0.31 & 4.50 & -342 & 702 & 755 \\
\hline & $2-\mathrm{SN}$ & 0.43 & 7.53 & 0.24 & -1.45 & 0.57 & 7.80 & 0.18 & 0.02 & - & - & -354 & 723 & 763 \\
\hline & $\mathrm{N}$ & - & 7.62 & 0.28 & - & - & - & - & - & - & - & -405 & 815 & 826 \\
\hline & $\mathrm{SEP}^{* * *}$ & - & 7.73 & 0.61 & 0.33 & 3.32 & - & - & - & - & - & -344 & 696 & 720 \\
\hline & $\mathrm{SN}$ & - & 7.62 & 0.28 & 0.00 & - & - & - & - & - & - & -405 & 817 & 834 \\
\hline \multirow{6}{*}{8} & $2-\mathrm{N}$ & 0.45 & 7.47 & 0.22 & 0.55 & 7.87 & 0.16 & - & - & - & - & -222 & 454 & 483 \\
\hline & 2-SEP & 0.08 & 7.24 & 0.63 & 0.14 & 2.38 & 0.92 & 7.83 & 0.63 & 0.26 & 2.89 & -217 & 451 & 504 \\
\hline & 2-SN & 0.45 & 7.47 & 0.22 & 0.01 & 0.55 & 7.86 & 0.16 & 0.03 & - & - & -222 & 458 & 499 \\
\hline & $\mathrm{N}$ & - & 7.69 & 0.27 & - & - & - & - & - & - & - & -306 & 617 & 628 \\
\hline & $\mathrm{SEP}^{* * *}$ & - & 7.86 & 0.70 & 0.29 & 2.61 & - & - & - & - & - & -219 & 447 & 470 \\
\hline & $\mathrm{SN}$ & - & 8.02 & 0.43 & -3.10 & - & - & - & - & - & - & -240 & 487 & 504 \\
\hline
\end{tabular}

Table 5: Fitted parameters for interval completion time distributions of Chicago marathon 2017 (* denotes distributions with lowest $\mathrm{AIC}$; ** denotes distributions with lowest BIC).

\subsubsection{New York city marathon}

It is clear from Table 2 that 2-SN and 2-SEP give the best approximations towards $\log t_{5 n}, n=$ $1,2, \ldots, 7$. The AIC of 2 -SN and 2 -SEP achieved in these intervals are significantly lower than that of other distributions, with differences larger than 10 units in most cases, which is considered 
strong evidence against other models according to Burnham and Anderson (2004). Figure 4 shows that during the first half of the marathon, slight bimodality is observed and that bimodality slowly disappears in time. This is caused by the decrease of peakedness of the faster running group, as a result of increasing variances and skewnesses, as shown in Figure 8. During the last interval of the marathon, bimodality is almost unobservable, distributions of both subpopulations can be modeled by a negatively skewed and platykurtic SEP.
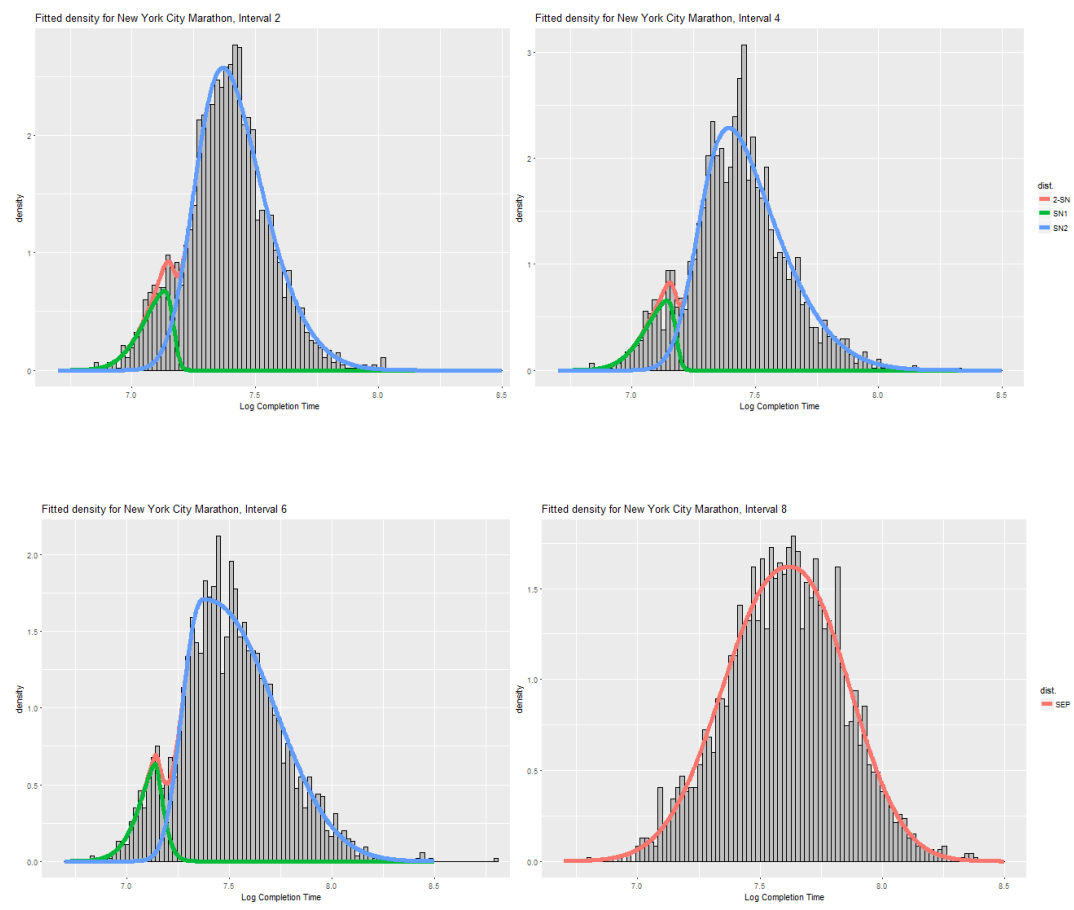

Figure 4: Fitted density for New York City marathon interval completion times.

Furthermore, after interval 4, the first four moments appear to follow some trend. Mean and variance of both groups are gradually increasing, skewness of the qualifier group is increasing and kurtosis of the non-qualifier group is decreasing. Overall, we can conclude that the 2-SEP is the most appropriate distribution modeling all intervals adequately.

\subsubsection{Boston marathon}

Table 3 shows that both 2-SEP and 2-SN continue to dominate other classes of distributions during the first half of the marathon, when negative skewness is significant for the qualifier group. As shown in Figure 5, negative skewness of the qualifier group becomes insignificant from interval 4, 2-N appears to fit as good as 2-SN in terms of likelihood but not as good as 2-SEP. This shows that skewness is no longer significant but kurtosis is still significantly different from the Gaussian distribution.

A possible explanation for the faster increase in skewness compared to that in New York city marathon is that, qualifiers in Boston marathon are in general slower than those in New York city 
marathon because of the slower qualifying time. The slower runner slows down at a faster rate with higher uncertainty. This can cause skewness to increase at a faster rate.
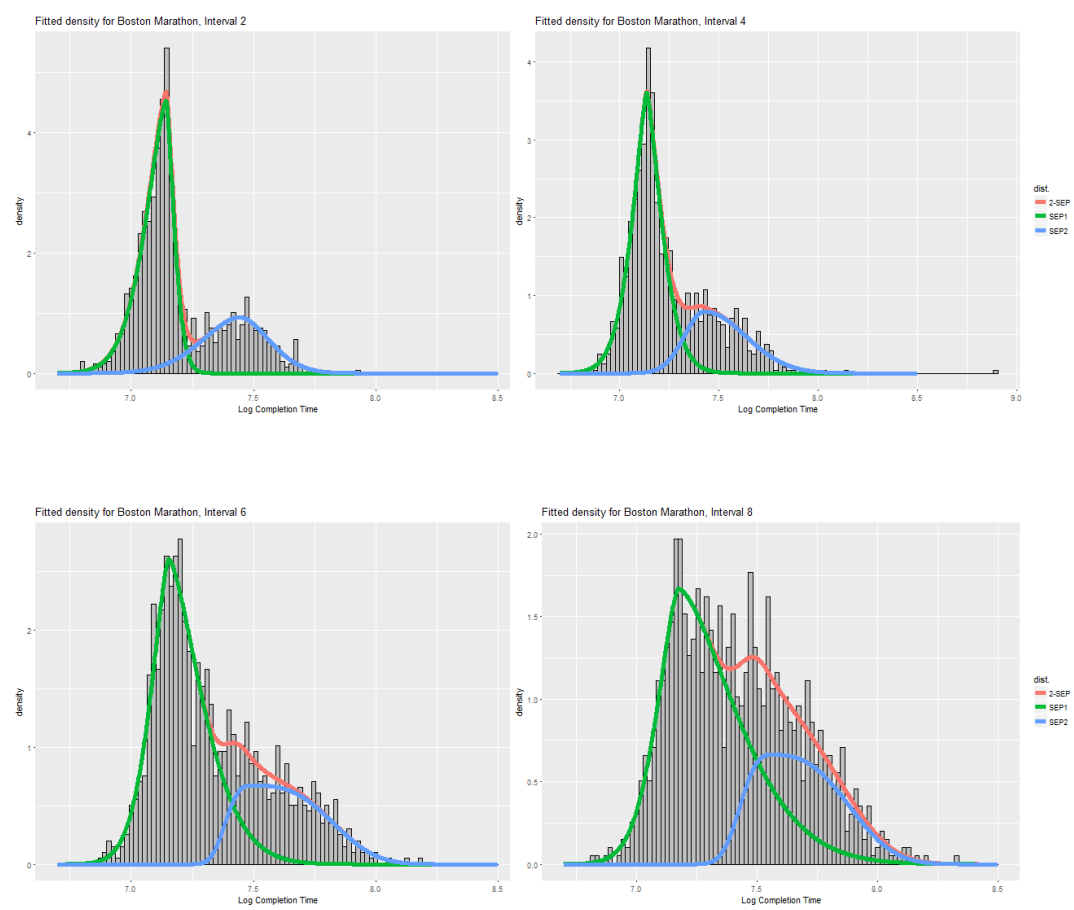

Figure 5: Fitted density for Boston marathon interval completion times.

Moreover, the first four moments after interval 4 appear to follow similar trends as observed in the New York city dataset, see Figure 8. The distribution for the non-qualifier group becomes more platykurtic with increasing variance over time especially in the last four intervals. We believe that this is the major reason why 2-SEP fits the dataset much better than other distributions in terms of likelihood.

\subsubsection{Berlin marathon}

For the Berlin marathon dataset, parameter estimates are shown in Table 4 . Some of the best fitted interval completion times distributions are plotted in Figure 6. The Berlin marathon has the strictest qualifying times among all four and less than $5 \%$ of runners finished faster than the qualifying time. Mixtures of distributions no longer have a significant edge over non mixture distributions as in the previous datasets analysed. Overall, both 2-SN and 2-SEP give similar fits for the dataset, because the kurtosis of the non-qualifier group is close to three during most intervals. Furthermore, similar trends of changing moments observed in New York and Boston marathons are noted after interval 4, see Figure 8. 

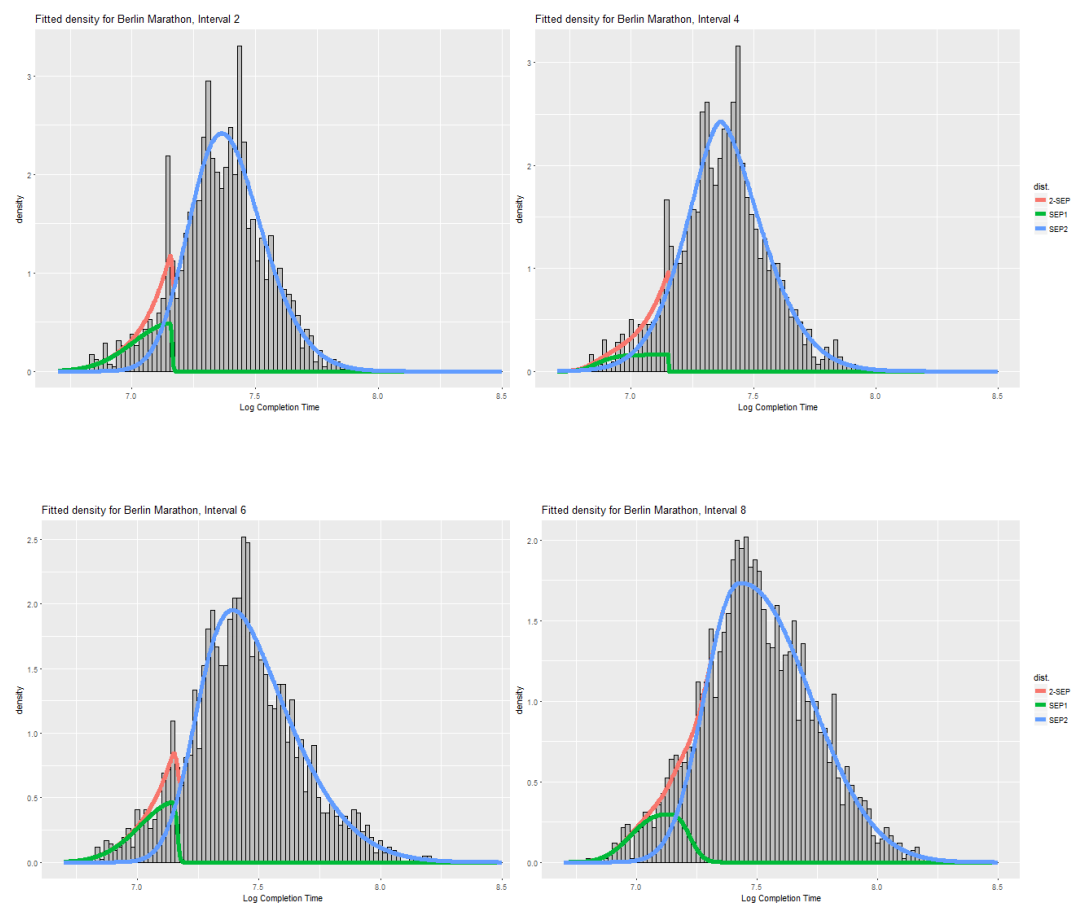

Figure 6: Fitted density for Berlin marathon interval completion times.

\subsubsection{Chicago marathon}

For Chicago marathon, estimates of the fitted parameters are shown in Table 5. 2-SEP fits better much better than all other distributions in the first six intervals, with differences in AIC larger than 10 mostly. As illustrated in Figure 7, one major reason that 2-SEP has an edge over 2-SN is the platykurtic shape of the non-qualifier group observed during early intervals of the marathon. Bimodality disappears in the last two stages, the distribution can be modeled adequately by a negatively skewed platykurtic SEP distribution. Again, the trend in changing moments observed after interval 4 is very similar to those observed in other datasets, see Figure 8. 

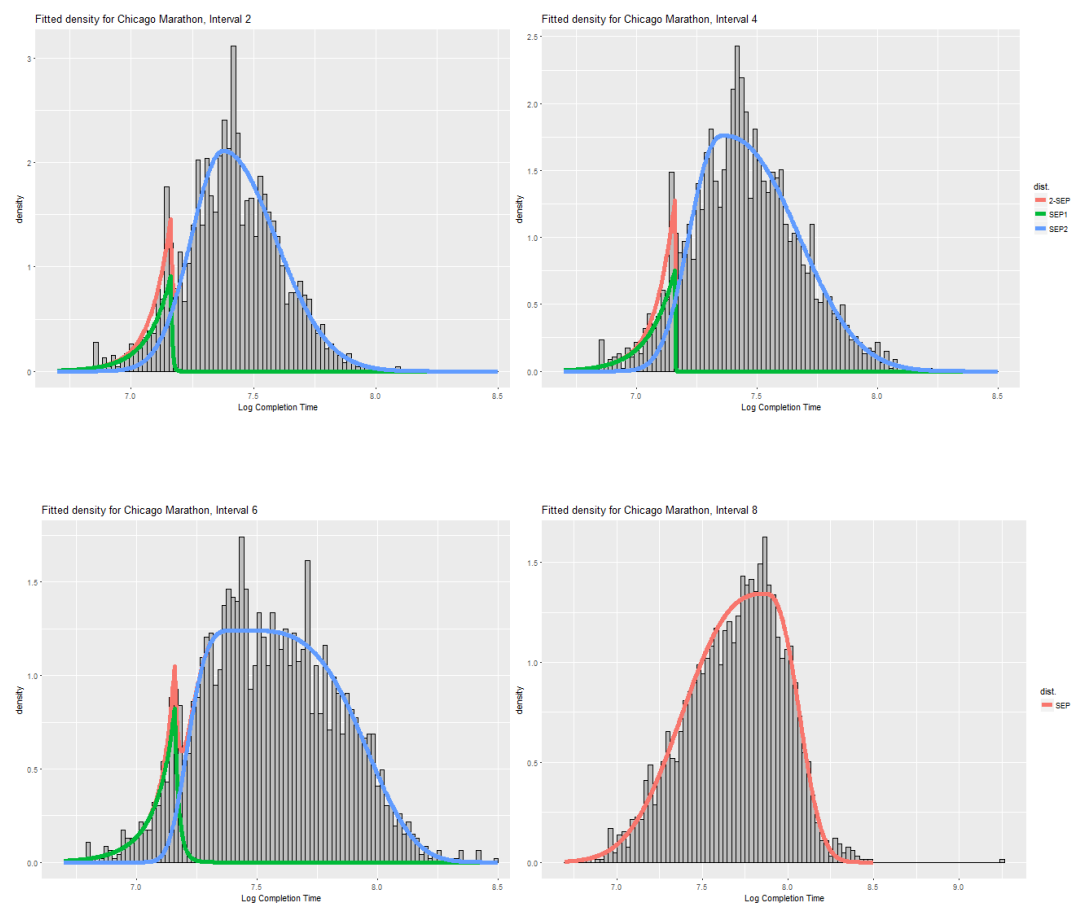

Figure 7: Fitted density for Chicago marathon interval completion times.

\subsubsection{Summary}

Overall, there are some properties that are common among all four datasets, which are shown in Figure 8. Both mean and variance show a clear increasing trend over the course of the marathon and they increase at a faster rate after interval 4 . We also see an increasing skewness trend for the qualifier groups after interval 4, and a decreasing trend of kurtosis for the non-qualifier groups also after interval 4. These results are coherent with our model explained earlier in this section - not only means and variances are changing over time, skewness and kurtosis are also changing. 

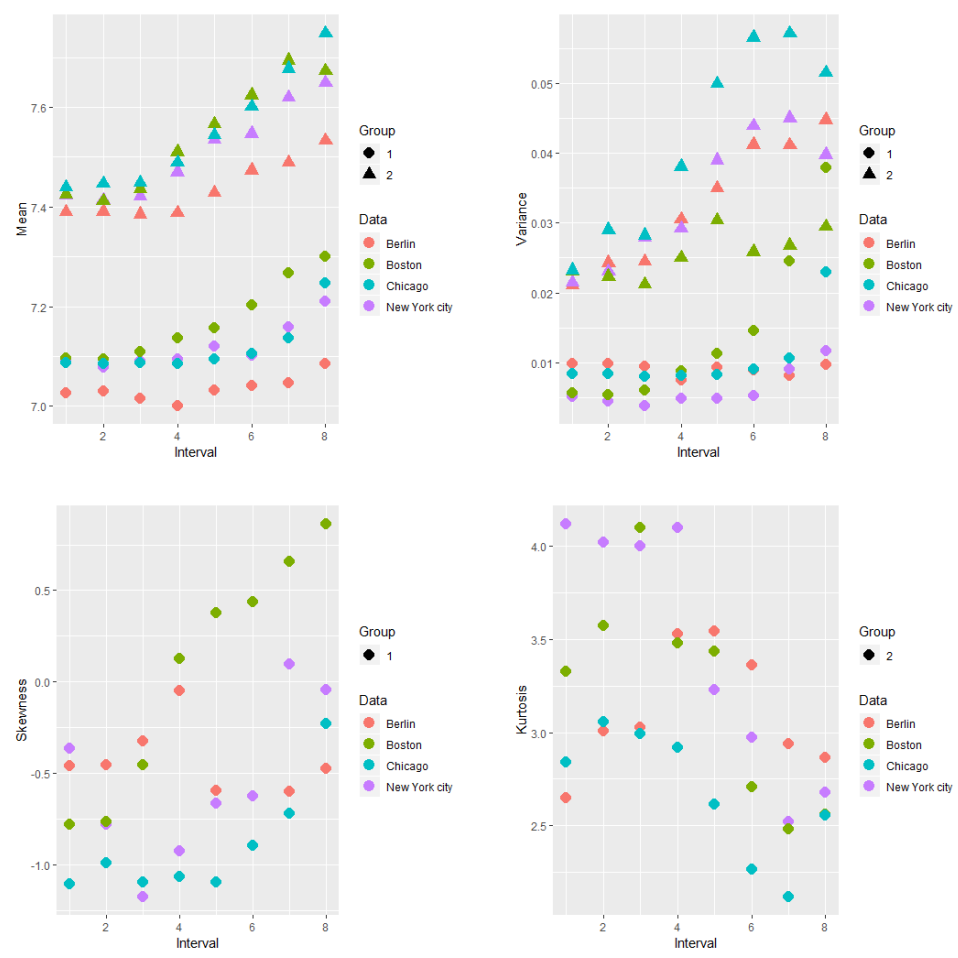

Figure 8: Moments of fitted distributions at each interval. Group 1 denotes the qualifier group. Group 2 denotes the non-qualifier group.

These trends in the first four moments after interval 4 suggest that the dynamic of marathon changes significantly after interval 4. A possible explanation is that, during the first half of the marathon, the majority of runners managed to attain relatively stable velocity, yielding relatively stable shapes of distributions. After the mark of 20 kilometers, more and more runners start to adjust their velocities. For the qualifier group, while the fastest runners remain at the same pace, the slower runners slow down, causing increases of the skewness, mean and variance of the qualifier group. For the non-qualifier group, many runners start to slow down. However, extremely slow interval completion times are not expected to be observed unless injuries are involved. For example, it is reasonable to assume that majority of healthy men aged from 25 to 29 can walk about 5 kilometers per hour. On the other hand, extremely fast interval completion times are also very rare at this stage after running 20 kilometers. As a result, the interval completion times of the non-qualifier group remain at very similar ranges after interval 4 , while mean and variance gradually increase, causing kurtosis to decrease.

\section{Model for log change of average velocity}

To further understand human running dynamics in a marathon, it is essential to recognise how human running velocities change from time to time. In this section, we develop a model to estimate properties of the change of average velocity given the present average velocity.

As shown in (3), we can write $\log v_{5 n+5}=\log v_{5 n}+g_{5 n}$, the change of shape from $\log v_{5 n}$ to $\log v_{5 n+5}$ can be described with the aid of coskewness and cokurtosis. Let $X$ and $Y$ be some 
dependent random variables. The skewness $S_{X+Y}$ and kurtosis $K_{X+Y}$ of $X+Y$ can be estimated from the following formulas:

$$
S_{X+Y}=\frac{\sigma_{X}^{3} S_{X}+3 \sigma_{X}^{2} \sigma_{Y} S(X, X, Y)+3 \sigma_{X} \sigma_{Y}^{2} S(X, Y, Y)+\sigma_{Y}^{3} S_{Y}}{\sigma_{X+Y}^{3}}
$$

and

$K_{X+Y}=\frac{\sigma_{X}^{4} K_{X}+4 \sigma_{X}^{3} \sigma_{Y} K(X, X, X, Y)+6 \sigma_{X}^{2} \sigma_{Y}^{2} K(X, X, Y, Y)+4 \sigma_{X} \sigma_{Y}^{3} K(X, Y, Y, Y)+\sigma_{Y}^{4} K_{Y}}{\sigma_{X+Y}^{4}}$,

where

$$
S(X, Y, Z)=\frac{E\{[X-E(X)][Y-E(Y)][Z-E(Z)]\}}{\sigma_{X} \sigma_{Y} \sigma_{Z}}
$$

and

$$
K_{A, B, C, D}=\frac{E\{[A-E(A)][B-E(B)][C-E(C)][D-E(D)]\}}{\sigma_{A} \sigma_{B} \sigma_{C} \sigma_{D}}
$$

are the coskewness and cokurtosis, respectively. In general, we can interpret $S(X, Y, Y), K(X, X, Y, Y)$ and $K(X, X, X, Y)$ in the following ways:

- $S(X, Y, Y)$ is positive if, $X$ has more positive deviations (not necessarily extreme) when $Y$ has extreme deviations;

- $S(X, Y, Y)$ is negative if, $X$ has more negative deviations (not necessarily extreme) when $Y$ has extreme deviations;

- $K(X, X, X, Y)$ is negative if, $X$ has positive extreme deviations when $Y$ has negative deviations (not necessarily extreme) or vice versa;

- $K(X, X, X, Y)$ is positive if, $X$ has positive extreme deviations when $Y$ has positive deviations (not necessarily extreme) or vice versa;

- $K(X, X, Y, Y)$ is large if, $X$ and $Y$ undergo extreme deviations at the same time. 


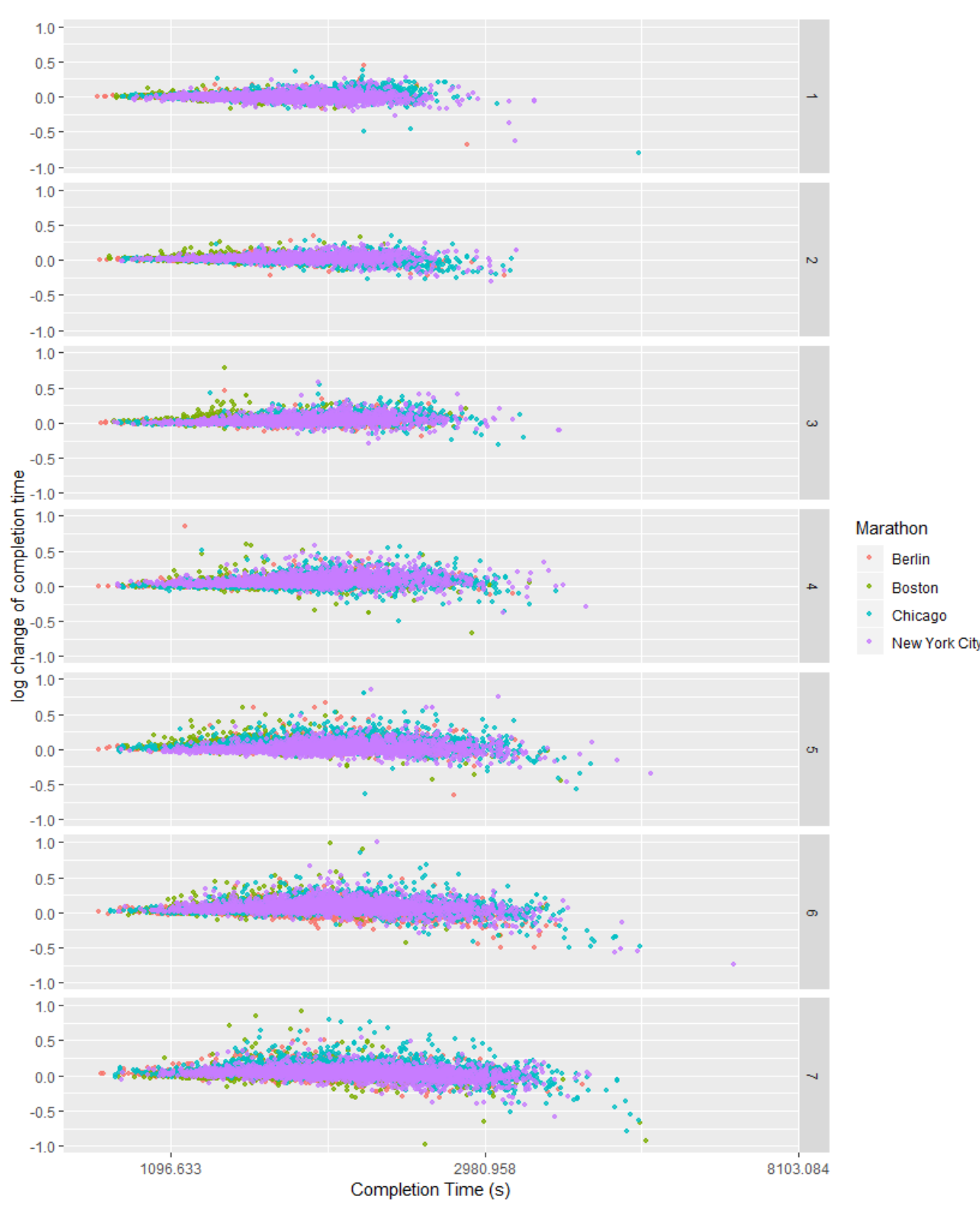

Figure 9: Log change of completion time against log completion time at intervals 1 to 7.

Since $g_{5 n}$ is the log change of average velocity, $-g_{5 n}$ describes the log change of interval completion time. Figure 9 illustrates the relationship between $\log t_{5 n}$ and $-g_{5 n}$ for each marathon. For all marathons, similar relationships mentioned in Section 2 can be observed. The same information can help us to understand the values of coskewness and cokurtosis.

We can see from Figure 9 how $S\left(\log t_{5 n},-g_{5 n},-g_{5 n}\right)$ and $S\left(\log t_{5 n}, \log t_{5 n},-g_{5 n}\right)$ vary over time. For example, we expect most $S\left(\log t_{5 n},-g_{5 n},-g_{5 n}\right)$ to be positive because extreme deviations of $-g_{5 n}$ happen mostly when $\log t_{5 n}$ is large. This captures the fact that slower runners are more unstable.

$S\left(\log t_{5 n}, \log t_{5 n},-g_{5 n}\right)$ are likely to be close to zero for the first half of the marathon because $-g_{5 n}$ were rather stable. During the later half of the marathon, more extreme negative deviations of $-g_{5 n}$ are observed for large $\log t_{5 n}$; however, many positive deviations of $-g_{5 n}$ are also observed for average $\log t_{5 n}$. As a result, the corresponding values of $S\left(\log t_{5 n}, \log t_{5 n},-g_{5 n}\right)$ are expected to be slightly negative. This captures the fact that runners become more unstable at later stages of the marathon and extreme speed ups are frequently observed among the slowest runners.

Similarly, $K\left(\log t_{5 n}, \log t_{5 n}, \log t_{5 n},-g_{5 n}\right)$ and $K\left(\log t_{5 n},-g_{5 n},-g_{5 n},-g_{5 n}\right)$ are expected to be 
close to zero or slightly positive during early stages, because the runners were rather stable. During the later stages, $K\left(\log t_{5 n},-g_{5 n},-g_{5 n},-g_{5 n}\right)$ is expected to be negative because $-g_{5 n}$ undergoes extreme negative deviations when $\log \left(\log t_{5 n}\right)$ is large.

During the early stage of the marathon, $K\left(\log t_{5 n}, \log t_{5 n}, \log t_{5 n},-g_{5 n}\right), K\left(\log t_{5 n},-g_{5 n},-g_{5 n},-g_{5 n}\right)$, $S\left(\log t_{5 n},-g_{5 n},-g_{5 n}\right)$ and $S\left(\log t_{5 n}, \log t_{5 n},-g_{5 n}\right)$ are expected to be close to zero or slightly positive, which would increase the value of third and fourth central moments of $t_{5 n+5}$. However, since skewness and kurtosis are standardized moments, and variance of $t_{5 n+5}$ is also increasing, the slight increases in central moments are unlikely to cause increases in skewness and kurtosis. As a result, both skewness and kurtosis are drawn closer to zero. During the later stages of the marathon, $S\left(\log t_{5 n}, \log t_{5 n},-g_{5 n}\right)$ is expected to be close to zero while $K\left(\log t_{5 n},-g_{5 n},-g_{5 n},-g_{5 n}\right)$ is expected to be slightly negative. In this case, according to (8) and (9), we expect that skewness will become closer to zero and kurtosis will decrease.

Overall, we can conclude that the log change of average velocity $g_{5 n}$ is correlated to $t_{5 n}$ in a significantly non-linear way. Some statistical properties observed are as follows: i) for the majority of runners, the variance of $g_{5 n}$ is higher for slower runners; ii) the distribution of $g_{5 n} \mid t_{5 n}$ appears to be skewed; iii) the distribution of $g_{5 n}$ has a high mean for the slowest runner during the later stages of the marathon.

We propose there exists two heterogeneous mechanisms of runners' velocity changes. The first one models velocity changes of runners who are running, i.e. runners with decreasing stamina. The second one models runners who are recovering, i.e. runners with recovering stamina. It is reasonable to assume that significant speed up is rare but significant slow down is common among runners in the first mechanism and vice versa in the second mechanism. Under such mechanisms, both models are skewed and there is a higher chance that slower runners are recovering than faster runners. From this information, we assume that the relationship between $\log v_{5 n}$ and $g_{5 n}$ can be explained by a mixture of generalized linear models with skewed errors. However, the distribution of $g_{5 n}$ is highly leptokurtic. Simple distributions like the skew normal distribution are not appropriate to describe the errors. At the same time, distributions like the SEP have such a complex density function that their fitting will be computationally expensive. To circumvent this, we use a mixture of three generalized linear models with skew normal errors. The extra model is used to compensate the shape misspecifications. The overall model we use is a mixture of three generalized linear models with skew normal errors and logit weight functions (3-SNR-Logit). The model is specified as follows:

$$
\begin{aligned}
f_{g}\left(g_{n} \mid t_{n}\right) & =\sum_{k=1}^{3} \omega_{k}\left(X, \beta_{0}\right) f\left(g_{n} ; X \beta_{1, k}, e^{X \beta_{2, k}}, X \beta_{3, k}\right), \\
\omega_{k}\left(X, \beta_{0}\right) & =\frac{\exp \left(X \beta_{0, k}\right)}{\sum_{i=1}^{3} \exp \left(X \beta_{0, i}\right)},
\end{aligned}
$$

where $f(\cdot)$ denotes the skew normal density function; $\beta_{0, k}=\left(\beta_{0, k, 1}, \beta_{0, k, 2}\right)^{T}$ are $2 \times 1$ matrices for $k=1, \ldots, 3$ and $\beta_{0,1}=(0,0)^{T} ; \beta_{0}=\left(\beta_{0,1}, \beta_{0,2}, \beta_{0,3}\right)$ denotes the parameters of the logistic regression weight function; $X=\left(1, \log t_{5 m}\right)$. Let $\beta_{k}=\left(\beta_{1, k}, \beta_{2, k}, \beta_{3, k}\right)$ and $\beta_{q, k}=\left(\beta_{q, k, 1}, \beta_{q, k, 2}\right)^{T}$ for $q, k=1,2,3$, where $k$ corresponds to the cluster of the model. Since the skew normal distribution is not in the exponential family, there is no standard procedure for parameter estimation. We use Markov Chain Monte Carlo (MCMC) to obtain the posterior distributions of the parameters $\beta_{0}$, $\beta_{1}, \beta_{2}, \beta_{3}$. 
The most efficient way of MCMC for finite mixture models is to treat group membership as a missing variable. We let $\boldsymbol{z}=\left(z_{0}, z_{1}, \ldots, z_{n}\right)$ denote the group membership of each data point. With the auxiliary variable $\boldsymbol{z}$, the posterior distributions will have simpler form and can be sampled more efficiently.

The likelihood function $p\left(\boldsymbol{g} \mid \boldsymbol{t}, \boldsymbol{z} ; \beta_{1}, \beta_{2}, \beta_{3}\right)$ given $\boldsymbol{t}, \boldsymbol{z}$ can be presented as follows:

$$
\begin{aligned}
& p\left(\boldsymbol{g} \mid \boldsymbol{t}, \boldsymbol{z} ; \beta_{1}, \beta_{2}, \beta_{3}\right)=\prod_{i=1}^{n} f_{g}\left(g_{i} \mid t_{i}, z_{i} ; \beta_{1, z_{i}}, \beta_{2, z_{i}}, \beta_{3, z_{i}}\right), \\
& f_{g}\left(g_{i} \mid t_{i}, z_{i} ; \beta_{1, z_{i}}, \beta_{2, z_{i}}, \beta_{3, z_{i}}\right)=f\left(g_{i} ; X \beta_{1, z_{i}}, e^{\left.X \beta_{2, z_{i}}, X \beta_{3, z_{i}}\right) .}\right.
\end{aligned}
$$

Then we have the following:

$$
\begin{aligned}
& P\left(z_{i} \mid g_{i}, t_{i} ; \beta_{1}, \beta_{2}, \beta_{3}\right)=\frac{f_{g}\left(g_{i} \mid t_{i}, z_{i} ; \beta_{1, z_{i}}, \beta_{2, z_{i}}, \beta_{3, z_{i}}\right)}{\sum_{k=1}^{3} f_{g}\left(g_{i} \mid t_{i}, k ; \beta_{1, k}, \beta_{2, k}, \beta_{3, k}\right)}, \\
& \pi\left(\beta_{0, k} \mid \boldsymbol{z}, \boldsymbol{t}\right) \propto \prod_{i=1}^{n} \omega_{i}\left(X, \beta_{0}\right) \pi\left(\beta_{0, k}\right), \\
& \pi\left(\beta_{k} \mid \boldsymbol{z}, \boldsymbol{t}, \boldsymbol{g}\right) \propto \prod_{i: z_{i}=k} f_{g}\left(g_{i} \mid t_{i}, k ; \beta_{1, k}, \beta_{2, k}, \beta_{3, k}\right) \pi\left(\beta_{1, k}\right) \pi\left(\beta_{2, k}\right) \pi\left(\beta_{3, k}\right) .
\end{aligned}
$$

We use Hamiltonian Monte Carlo (HMC) (Duane et al. 1987) to sample from both posterior distributions of $\beta_{k}$ and $\beta_{0}$. HMC is used because it is more efficient than many other popular MCMC algorithms such as Random-walk Metropolis-Hastings (RWM) sampler. HMC has a higher rate of convergence and can more efficiently explore the sample space if appropriately tuned (see Neal (2012) for details). HMC is most effective when all the parameters are updated simultaneously. Unfortunately, in a mixture model problem, simultaneous update is not possible under a latent variable approach. As a result, the algorithm would still generate autocorrelated samples.

For illustration, 50,000 data points of log average velocity changes from interval 7 to interval 8 and average velocities at interval 7 were sub-sampled randomly from the full dataset. To increase computational efficiency, both variables were normalized to have zero means and unit variances. 8,000 MCMC samples were generated by the routine described below with the following specifications: uniform (improper) priors are used for all parameters; for $\beta_{0}$, leap-frog with step number of 100 and a random step size drawn uniformly from $U(0.01,0.05)$; for $\beta_{k}$, leap-frog with step number of 50 and a random step size drawn uniformly from $U(0.001,0.01)$.

The MCMC algorithm is as follows:

1. Set initial parameters $\beta_{0}, \beta_{1}, \beta_{2}, \beta_{3}$;

2. Sample membership weight $\boldsymbol{z}$ from (16);

3. Update $\beta_{0}$ using posterior distribution from (17) using Hamiltonian dynamics;

4. Update $\beta_{k}$ using posterior distribution from (18) using Hamiltonian dynamics;

5. Repeat step 2 to step 4 until a desirable number of iterations has been completed. 
The first 3,000 samples were burnt and the remaining 5,000 samples were thinned ten times to reduce autocorrelation. Figure 12 shows that autocorrelations in $\beta_{0}, \beta_{1}$ and the variance parts of $\beta_{2}$ and $\beta_{3}$ are still significant after thinning. As a result, the estimated posterior distributions may be slightly biased, see Figure 11. Figure 10 plots the estimated $\log g_{35}$ given $v_{35}$ using the sample generated. When compared to the actual $\log g_{35}$, the simulated $\log g_{35}$ quite closely resembles the actual relationship, with group 1 representing velocity changes of the slowest runners. Both group 2 and group 3 describe the velocity changes distribution of the vast majority of runners. As mentioned the conditional distribution of the log change of velocity is leptokurtic, such shape can be attained by having a mixture of more spread out distribution (group 2) and a less spread out distribution (group 3). Overall, the fitted model closely resembles the actual relationship between $g_{35}$ and $v_{35}$.

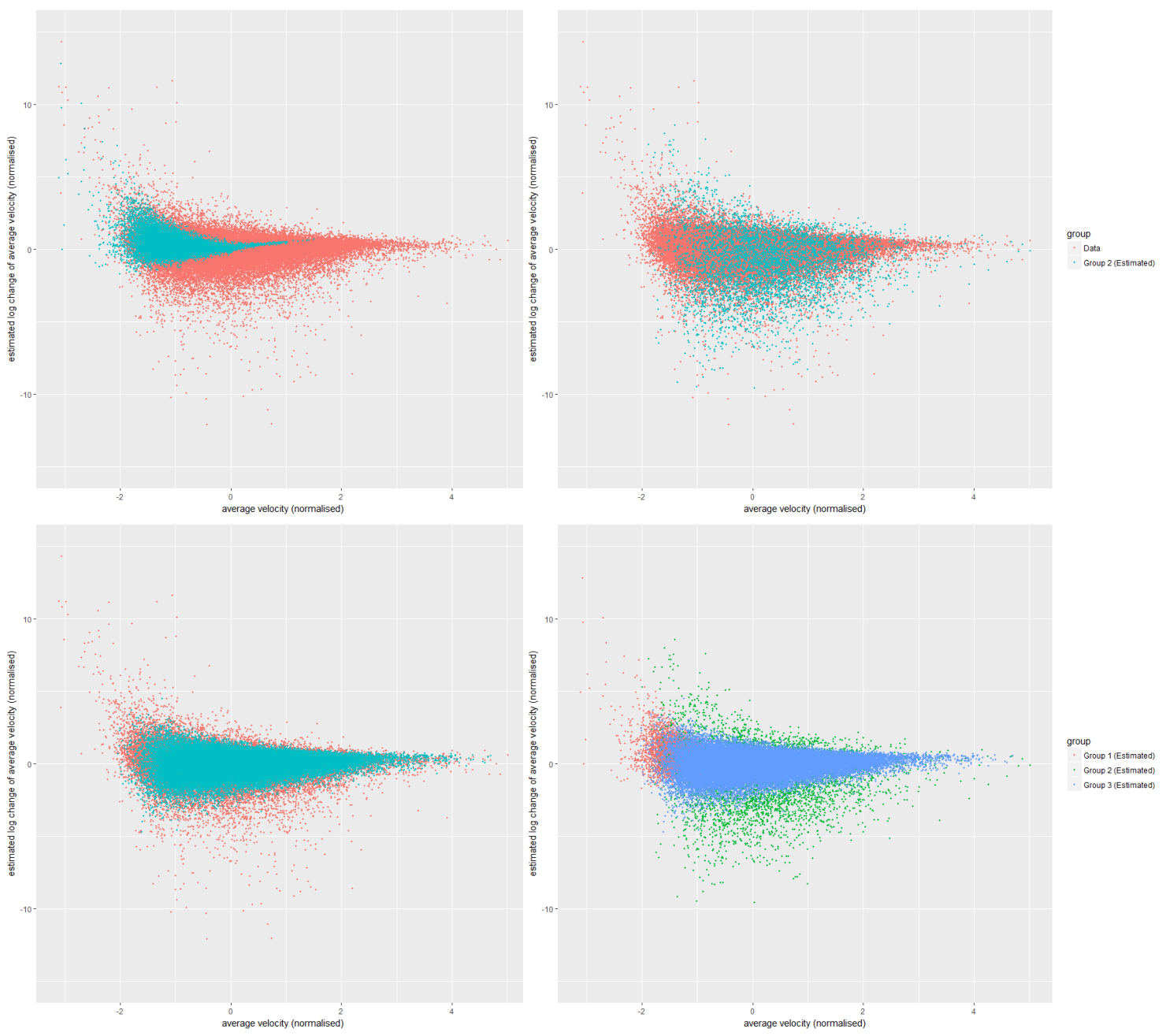

Figure 10: Simulated velocity changes. 

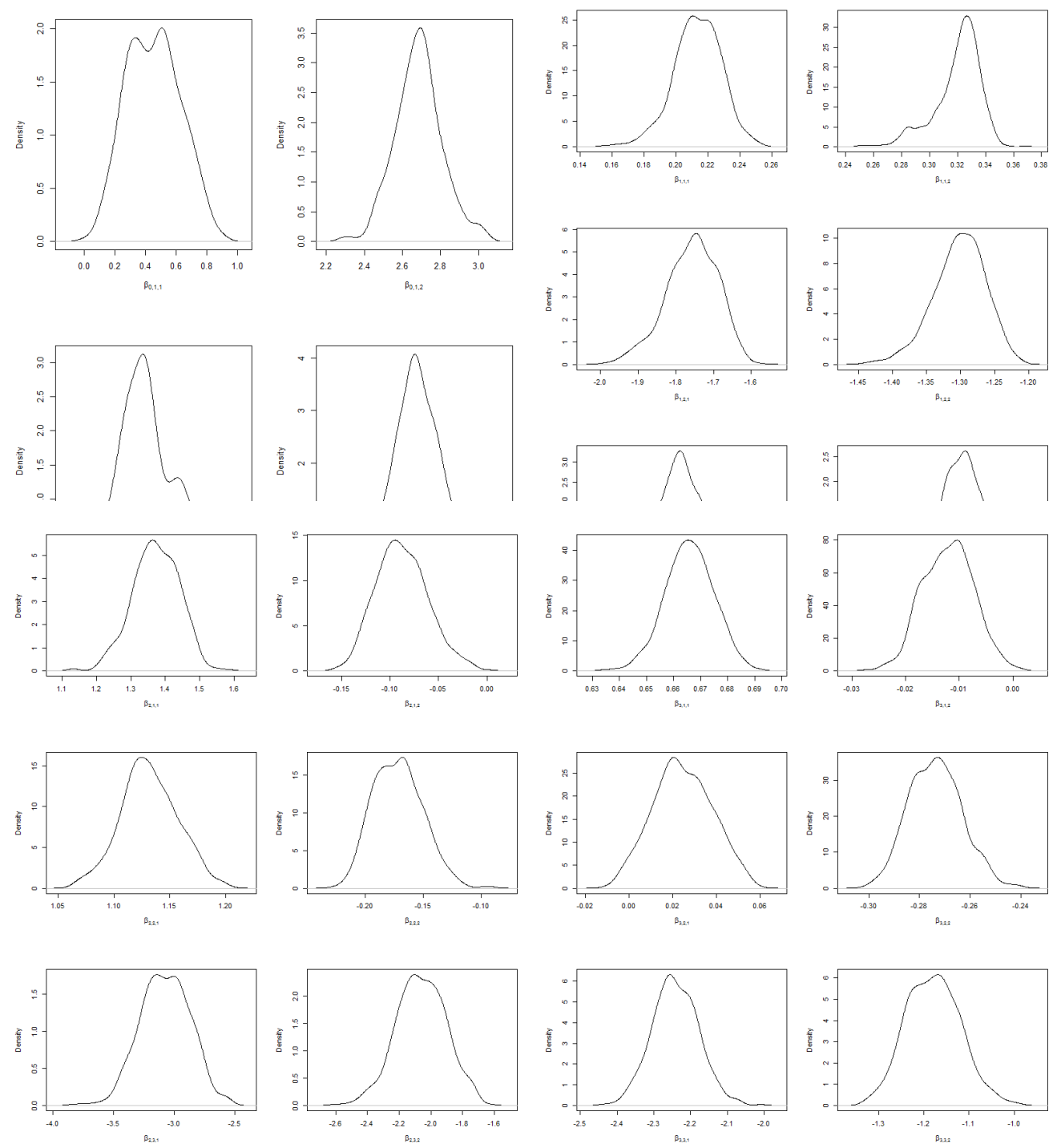

Figure 11: Posterior distributions from 500 MCMC samples. 

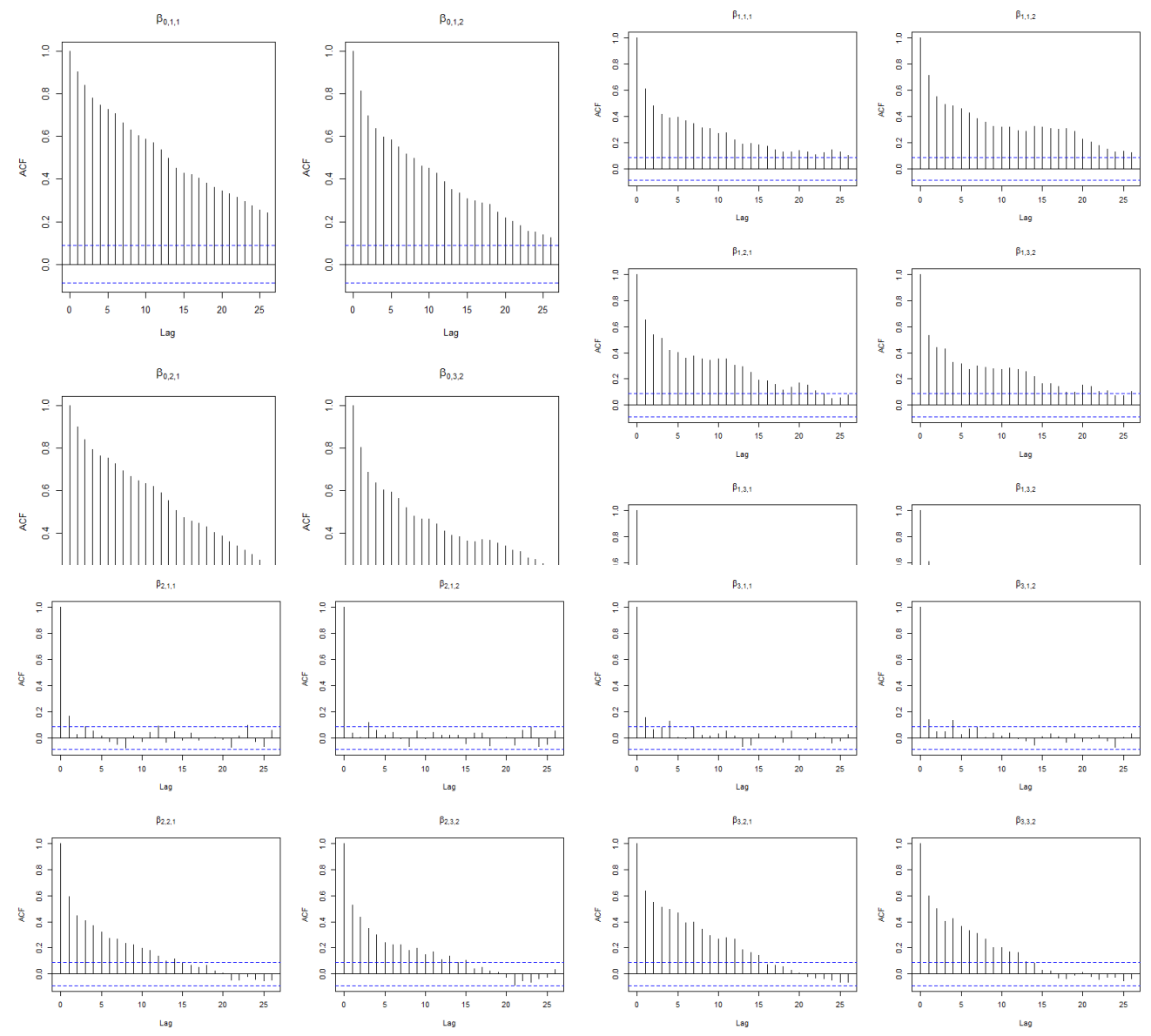

$\beta_{2,3}$
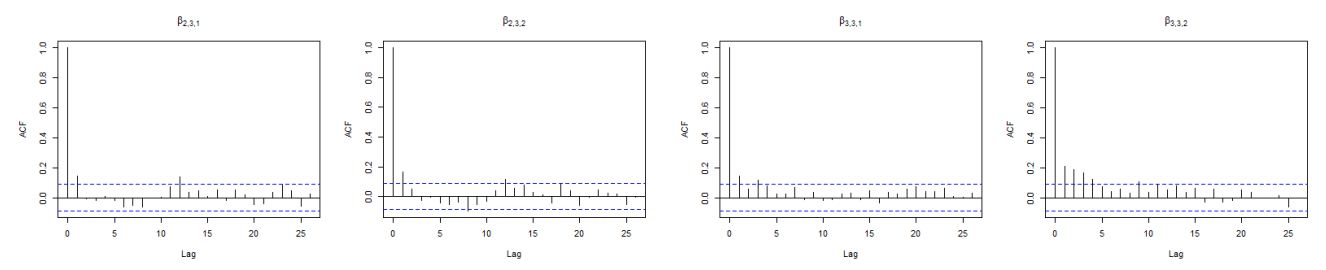

Figure 12: Autocorrelation plots from 500 MCMC samples.

\section{Conclusions}

In this paper, we have developed models describing runners' dynamics in a marathon. Empirical evidence has been presented to support our models. We have demonstrated the effectiveness of the SEP to describe distribution of log completion times in a marathon. By comparing the model to other models in terms of AIC, we find that 2-SEP distributions give significantly better fits than other distributions considered for most datasets. We have also analysed the process of how runners' velocity changes. We found that changes of velocity are highly dependent on current velocity, which can be explained by two heterogeneous mechanisms. The model we proposed (3-SNR-Logit) has been shown to be effective in estimating relationship between log change of average velocity and average velocity. We have not seen a model of similar type as 3-SNR-Logit, i.e. a finite mixture of generalized linear models with varying weights, being used in any previous research. We have shown that an MCMC algorithm can be used to generate posterior samples from this type of model. We believe the model is not only useful in explaining velocity changes in a marathon, but also in other 
processes involving human behaviours/decisions; as human behaviours are usually too complex to be explained by exponential family distributions; and human changes usually happen abruptly, e.g. taking walk breaks in a marathon. With the aid of finite mixture models with varying weights, such mechanisms can be explained more fully.

\section{Acknowledgments}

The authors would like to thank the Editor and the two referees for careful reading and comments which greatly improved the paper.

\section{References}

[1] Akaike, H. (1974). A new look at the statistical model identification. IEEE Transactions on Automatic Control, 19, 716-723.

[2] Alvarez-Ramirez, J. and Rodriguez, E. (2006). Scaling properties of marathon races. Physica A: Statistical Mechanics and Its Applications, 365, 509-520.

[3] Alvarez-Ramirez, J., Rodriguez, E. and Dagdug, L. (2007) Time-correlations in marathon arrival sequences. Physica A: Statistical Mechanics and Its Applications, 380, 447-454.

[4] Burnham, K. P. and Anderson, D. R. (2004). Multimodel inference: Understanding AIC and BIC in model selection. Sociological Methods and Research, 33, 261-304.

[5] Dempster, A. P., Laird, N. M. and Rubin, D. B. (1977). Maximum likelihood from incomplete data via the EM algorithm. Journal of the Royal Statistical Society, B, 39, 1-38.

[6] Duane, S., Kennedy, A. D., Pendleton, B.J. and Roweth, D. (1987). Hybrid monte carlo. Physics letters, B, 195, 216-222.

[7] Lehto, N. (2016). Effects of age on marathon finishing time among male amateur runners in Stockholm marathon 1979-2014. Journal of Sport and Health Science, 5, 349-354.

[8] Lin, Z. and Meng, F. (2018). Empirical analysis on the runners' velocity distribution in city marathons. Physica A: Statistical Mechanics and Its Applications, 490, 533-541.

[9] Neal, R. M. (2012). Bayesian Learning for Neural Networks. Springer, Berlin.

[10] Rodriguez, E., Espinosa-Paredes, G. and Alvarez-Ramirez, J. (2014). Convection-diffusion effects in marathon race dynamics. Physica A: Statistical Mechanics and Its Applications, 393, 498-507.

[11] Schwarz, G. E. (1978). Estimating the dimension of a model. Annals of Statistics, 6, 461-464.

[12] Zhu, D. and Zinde-Walsh, V. (2009). Properties and estimation of asymmetric exponential power distribution. Journal of Econometrics, 148, 86-99. 